\title{
MyD88 in donor bone marrow cells is critical for protection from acute intestinal graft-vs.-host disease
}

\author{
J-Y Lim ${ }^{1}$, Y-K Lee ${ }^{2}$, S-E Lee ${ }^{1}$, J-M Ju², K-S Eom ${ }^{1}$, Y-J Kim ${ }^{1}$, N-G Chung ${ }^{3}$, DC Jeong ${ }^{3}$, G Park ${ }^{4}$, \\ EY Choi ${ }^{2}$ and C-K Min ${ }^{1}$
}

To understand the role of myeloid differentiation factor 88 (MyD88) expressed by donor bone marrow (BM) in the pathophysiology of graft-vs.-host disease (GVHD), we investigated the effects of transplantation of MyD88-deficient T cell-depleted BM (MyD88KO TCD-BM) on the severity of GVHD. Transplantation with MyD88KO TCD-BM aggravated GVHD; serious gut damage was evident, with high infiltration of T cells into the intestines of recipients and markedly reduced expansion of $\mathrm{CD}_{11 \mathrm{~b}}{ }^{+} \mathrm{Gr}-1^{+}$myeloid-derived suppressor cells (MDSCs). MDSCs from MyD88KO mice were defective in inducing donor T-cell apoptosis and inhibiting T-cell proliferation. Supplementation of transplanted mice with MDSCs from wild-type mice, but not MyD88KO mice, attenuated GVHD severity with reduced intestinal T-cell infiltration in MyD88KO TCD-BM recipients. Pretreatment of BM donors with lipopolysaccharide to increase MDSC levels and MyD88 transcription in the TCD-BM transplant alleviated GVHD severity and intestinal T-cell infiltration. The T cell/MDSC ratios were correlated with intestinal GVHD severity in both animal models and human patients. This study indicates that MyD88-dependent MDSC expansion from donor BM is critical for protection against fatal intestinal GVHD.

\section{INTRODUCTION}

Allogeneic hematopoietic stem cell transplantation (alloHSCT) is an important therapeutic modality used to treat malignancies of hematopoietic origin, such as leukemia and lymphoma. However, development of graft-vs.-host disease (GVHD) causes non-relapse mortality and substantial morbidity of recipients. ${ }^{1}$ Gastrointestinal tract damage initiated during pre-conditioning is a principal pathological cause of mortality from GVHD. ${ }^{2}$ Gastrointestinal tract damage during GVHD exposes host epithelial and immune cells to the products of dying (eukaryotic) cells and commensal bacteria (such as lipopolysaccharides (LPS) and flagellin), which trigger the action of the toll-like receptor (TLR). TLRs are expressed by various cell types, not only on innate macrophage and dendritic cells but also on adaptive $\mathrm{T}$ and $\mathrm{B}$ cells, ${ }^{3}$ and non-hematopoietic epithelial cells. ${ }^{4}$ Myeloid differentiation factor 88 (MyD88), a major adaptor mediating TLR signaling, is also known to deliver pro-inflammatory signals. ${ }^{5}$ However, MyD88-signaling does not always cause inflammation, as shown by the fact that more severe inflammatory bowel disease is induced in MyD88deficient (MyD88KO) than wild-type (WT) mice. ${ }^{6}$

The role of MyD88 signaling in GVHD has not been investigated intensively, but some studies have produced relevant (although controversial) results. TLR-mediated signaling has been considered to contribute to initiation of GVHD via activation of antigen-presenting cells (APCs) that enhance T-cell activation. ${ }^{7,8}$ Linkage of a TLR4 polymorphism to GVHD severity, ${ }^{9}$ and aggravation of GVHD lethality after treatment of GVHD hosts with a TLR9 agonist ${ }^{10}$ suggested that suppression of TLR signaling would improve GVHD treatment. The reduced severity of intestinal GVHD in MyD88KO hosts ${ }^{4}$ is consistent with this view. However, despite the significance of host APCs in the initiation of GVHD, ${ }^{11}$ it has been reported that MyD88-deficiency specifically in host APCs did not ameliorate GVHD at all, ${ }^{12}$ suggesting that any role of MyD88 in GVHD might not be limited to APC activation, and that signaling by MyD88 in other types of hematopoietic cells, or epithelial cells could be involved in GVHD pathogenesis.

${ }^{1}$ Departments of Internal Medicine, Seoul St. Mary's Hospital, The Catholic University of Korea, Seoul, Korea. ${ }^{2}$ Department of Biomedical Sciences, Seoul National University College of Medicine, Seoul, Korea. ${ }^{3}$ Departments of Pediatrics, Seoul St. Mary's Hospital, The Catholic University of Korea, Seoul, Korea and ${ }^{4}$ Departments of Pathology, Seoul St. Mary's Hospital, The Catholic University of Korea, Seoul, Korea. Correspondence: EY Choi or C-K Min (eycii@snu.ac.kr or ckmin@catholic.ac.kr) 
Apart from its role in sensing inflammation, MyD88 has been implicated in control of the expansion of myeloid-derived suppressor cells (MDSCs), as shown by prevention of expansion of such cells in MyD88KO mice under conditions of sepsis. ${ }^{13}$ MDSCs are well known to effectively suppress T-cell responses both in tumor models and patients, ${ }^{14-16}$ and share the common phenotypic markers $\mathrm{CD}_{11 \mathrm{~b}}{ }^{+}$(Mac-1) and Gr- $1^{+}$(Ly6G/Ly6C) in mice. ${ }^{17}$ In work on murine alloHSCT, several studies have revealed expansion of MDSCs and associations between the proportions of such cells and reduced GVHD mortality. ${ }^{18,19}$ However, any relationship between MyD88 signaling and MDSC expansion in GVHD has been seldom investigated.

To explore the contribution of MyD88 expressed by donor bone marrow (BM) cells to development of GVHD, we induced GVHD using T-cell-depleted BM (TCD-BM) isolated from MyD88KO C57BL/6 (B6) mice and T cells isolated from WT B6 mice. We sought to determine the effect of loss of MyD88 expression by donor TCD-BM on the development of intestinal GVHD. Our results will show that MyD88 signaling in donor $\mathrm{BM}$ cells has a protective role via enhancing the expansion and immunosuppressive function of MDSCs derived from the donor TCD-BM. Our results also reveal the clinical relevance of the relative abundance of MDSCs compared with $\mathrm{T}$ cells in predicting the development of severe intestinal GVHD after experimental and clinical myeloablative allo-HSCT.

\section{RESULTS}

Allogeneic recipients of MyD88KO donor TCD-BM exhibit higher mortality and more severe intestinal damage

To evaluate the impact of the absence of MyD88 signaling in donor TCD-BM (the non-T-cell compartment) on GVHD progression, we induced acute GVHD in lethally irradiated B6D2F1 (F1) mice by injecting $1 \times 10^{6}$ T cells purified from WT B6 mice together with TCD-BM isolated from MyD88KO B6 mice (the major histocompatibility complex- and minor histocompatibility antigen (miHA)-mismatched model). Control GVHD hosts received T cells and TCD-BM both isolated from WT B6 mice. A dose of $1 \times 10^{6} \mathrm{~T}$ cells was chosen to induce mild acute GVHD, ${ }^{20}$ to allow morbidity to be compared between the two types of GVHD hosts that had received TCDBM from different donors over a long period of time.

Unexpectedly, all the recipients of MyD88KO TCD-BM died within 20 days (Figure 1a; the median survival time was 15.0 days) and showed high clinical GVHD scores (left, Figure 1b) with severe weight loss (right, Figure 1b) and serious diarrhea, whereas the recipients of WT TCD-BM did not exhibit such early lethality (median survival time: 62.5 days). In the allogeneic F1 recipients of MyD88KO TCD-BM only (lack of T-cell co-transplantation), myeloid $\left(\mathrm{CD} 11 \mathrm{~b}^{+}\right)$and $\mathrm{B}$ $\left(\mathrm{B} 220^{+}\right)$cells of donor origin $\left(\mathrm{H}-2^{\mathrm{d}}\right.$-negative) were evident in the BM on day 13 post transplantation (Supplementary Figure S1a online), indicating successful engraftment and hematopoiesis of transplanted MyD88KO TCD-BM. Syngeneic B6 recipients of MyD88KO TCD-BM and WT T cells exhibited good survival, whereas the total body irradiation-only controls (without BM transplantation) died within 8 days (Figure 1a). Therefore, the drastic mortality of allogeneic F1 recipients of MyD88KO TCD-BM plus WT T cells was ascribed to the alloreactivity of transplanted $\mathrm{T}$ cells.

Gross examination of isolated organs revealed more prominent lengthening of the small intestines (SI), a sign of presence of intestinal GVHD, in the allogeneic recipients (allo $\mathrm{KO}$ ) of MyD88KO TCD-BM and WT T cells than in those (allo WT) of WT TCD-BM and WT T cells (Figure 1c). Consistently, histological examination revealed that total epithelial denudation (grade 4) was more marked, and severe GVHD lesions ( $\geqslant$ grade 3 ) were more prevalent in the SI of the MyD88KO TCD-BM recipients than in those of the WT recipients (lengths of the lesions ( $\geqslant$ grade 3 ) as percentages of the entire SI lengths were $81.7 \pm 6.2$ in the case of the MyD88KO TCD-BM recipients and $53.3 \pm 16.9$ in the case of WT recipients; $P=0.46$, Figure 1d). These findings showed that lack of MyD88 expression in the non-T-cell compartment derived from donor BM aggravated the severity of GVHD, suggesting that the GVHD aggravation and enhanced mortality in the recipients of MyD88KO TCD-BM correlate with increases in intestinal damage.

\section{Donor T cells heavily infiltrate the small intestine of allogeneic recipients of MyD88KO TCD-BM}

In the allogeneic recipients, irrespective of donor $\mathrm{BM}$ origin (MyD88KO or WT), all the leukocytes infiltrating the GVHD target organs were of donor type (Supplementary Figure S1b). To more clearly compare the extent of tissue infiltration by donor T cells between the two groups of GVHD hosts, T cells isolated from WT congenic B6.Ly-5a B6 mice $\left(\mathrm{CD} 45.1^{+}\right)$were used to induce GVHD. The proportions of CD45.1 ${ }^{+} \mathrm{T}$ cells in tissue-infiltrating leukocytes were higher in the spleens, mesenteric lymph nodes (MLN), and SI of recipients of MyD88KO TCD-BM than in those of WT TCD-BM, because CD45.1 ${ }^{-}$non-T-cell compartments did not become normally expanded in the former (Figure 2a,b). However, the absolute numbers of infiltrating CD45.1 ${ }^{+} \mathrm{T}$ cells were lower in the spleens of the recipients of MyD88KO TCD-BM than in those of WT TCD-BM (Figure 2b), because the total cellularity levels were lower in the formers (data not shown). Nevertheless, however, the absolute numbers of CD $45.1^{+} \mathrm{T}$ cells infiltrating the intestine were significantly higher in the formers because of their high intestinal cellularity. Such organ-dependent differences were evident when the levels of both $\mathrm{CD}^{+}{ }^{+}$and $\mathrm{CD}^{+}$cell subtypes were examined (Figure 2c). CD $45.1^{+}$cells were more abundant in the mucosa of SI of MyD88KO TCD$\mathrm{BM}$ recipients than in those of WT controls by confocal microscopic analyses (Figure 2d). In support of these findings, the proportions of cells expressing CCR9, which is associated with T-cell migration to the intestinal mucosa, ${ }^{21}$ among the carboxyfluorescein succinimidyl ester (CFSE)-low alloreactive $\mathrm{CD}^{+} \mathrm{T}$ cells were higher in the spleens of MyD88KO TCD$\mathrm{BM}$ recipients than in those of the $\mathrm{WT}$ control group (Figure 2e): The CFSE-low cells were identified on the basis of the CFSE-dilution profiles of $\mathrm{T}$ cells under 


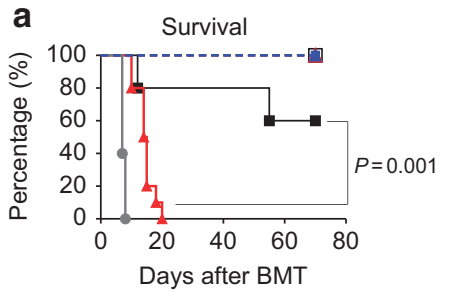

C
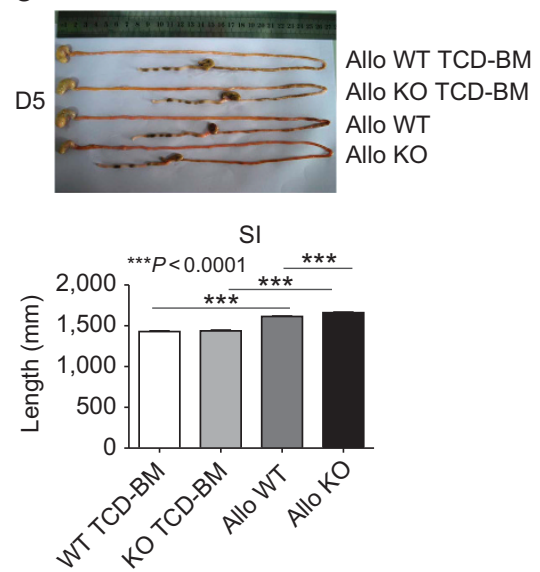

b

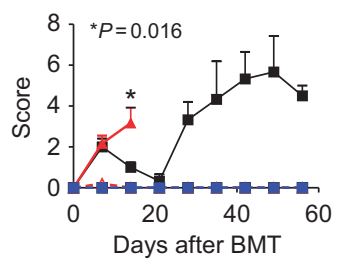

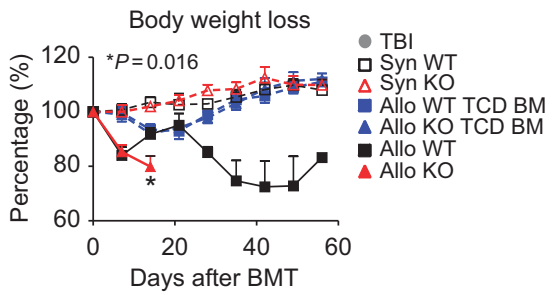

d

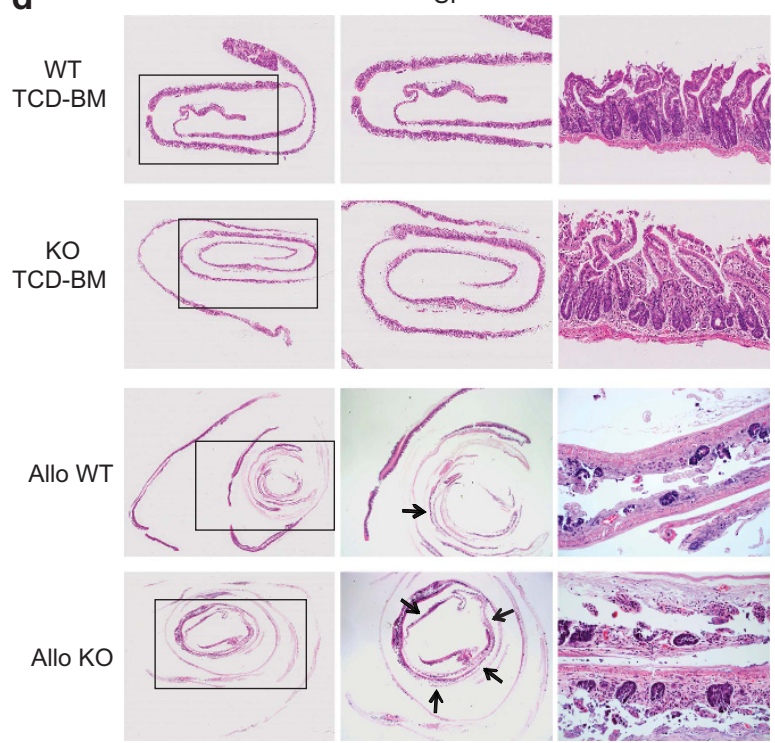

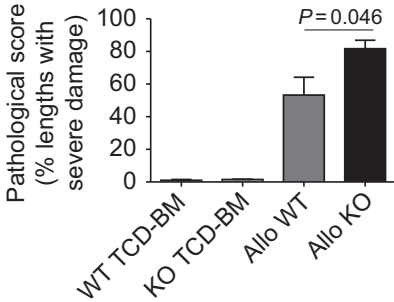

Figure 1 Allogeneic recipients of MyD88KO donor TCD-BM exhibit severe GVHD morbidity and mortality with intestinal damage. Lethally irradiated F1 recipients were given $5 \times 10^{6}$ WT or MyD88KO TCD-BM cells plus $1 \times 10^{6}$ purified WT T cells from allogeneic B6 donors $(n=10$ in each group, allo WT and allo KO). B6 animals were irradiated and transplanted with $5 \times 10^{6}$ WT or MyD88KO TCD-BM cells plus $1 \times 10^{6}$ purified WT T cells from syngeneic B6 donors ( $n=5$ in each group, syn WT and syn KO). Control mice were given only TCD-BM from WT or MyD88KO mice (thus without co-transplantation of B6 T cells; $n=10$ in each group, allo WT TCD-BM and allo KO TCD-BM), or were not given TCD-BM (total body irradiation (TBI), $n=5$ ) after the irradiation. (a) Kaplan-Meier survival curve of the transplanted mice. Percent survival after transplantation, allo WT vs. allo KO by the Wilcoxon rank-sum test. (b) Corresponding GVHD scores (left) and weight loss (right). Animals were assessed weekly for weight loss and clinical severity, allo WT vs. allo KO by the Mann-Whitney U-test. Data are presented as means \pm s.e.m. and are representative of duplicate experiments. (c) Whole gut was isolated from nonGVHD controls without T cells (allo WT TCD-BM and allo KO TCD-BM) and allogeneic recipients (allo WT and allo KO) on day 5. The severity of gut damage was grossly compared and representative animals are shown. The lengths of the small intestines (SI) are presented as means \pm s.e.m. (d) The extent of epithelial injury of the SI was evaluated. Areas of grade $\geqslant 3$ were classified as severely affected (arrows), and the proportions of such severe SI lesions were compared among different groups of transplantation. Representative pathological findings in severe lesions are indicated by arrows. The mid-panel figures $(\times 12.5)$ are the areas within the black squares of the left figures and the right figures $(\times 200)$ are representative of the severe lesions. The extent of pathological damage to SI was evaluated using the semi-quantitative scoring system described in Methods section, in all the transplantation groups ( $n=5$ in each group). Lengths of the lesions ( $\geqslant$ grade 3 ) were plotted as percentages of the entire SI lengths, ${ }^{39}$ and the values were denoted as pathological scores and compared among the different groups. Data are presented as means \pm s.e.m. Data shown in c andd are representative of two independent experiments. BMT, bone marrow transplantation; GVHD, graft-vs.-host disease; KO, knockout; TCD-BM, T-cell depleted bone marrow; WT, wild type.

syngeneic conditions on day 5. Accordingly, CCL25 (CCR9ligand) was produced at higher levels in the intestine of the MyD88KO group than in that of the WT control group on day 7 post transplantation (Figure 2f). On the other hand, $\alpha 4 \beta 7$ expression on donor $\mathrm{T}$ cells and production of its ligand (MAdCAM-1) in the SI were similar between the two groups (Supplementary Figure S2a). Together, these results showed that recipients of MyD88KO TCD-BM experienced increased intestinal infiltration by activated $\mathrm{T}$ cells, compared with the recipients of WT TCD-BM. We suggest that this presence, in higher frequencies, of CCR9 ${ }^{+} \mathrm{CFSE}^{\text {low }} \mathrm{T}$ cells in the spleens explains the enhanced infiltration of $\mathrm{T}$ cells in the SIs of myD88KO group.
In addition, to examine whether $\mathrm{CD} 45.1^{+}$donor $\mathrm{T}$ cells in the spleens of the two different GVHD hosts would differ in their apoptosis or proliferation capacities, Annexin V-staining and BrdU-incorporation analyses were performed with $\mathrm{T}$ cells from the mice treated with BrdU for $4 \mathrm{~h}$ before the sacrifice on day 4 post transplantation, the period before migration of activated cells from secondary lymphoid organs to peripheral tissues. ${ }^{22}$ Although BrdU-incorporation by $\mathrm{T}$ cells during the $4 \mathrm{~h}$ was not significantly different between the two groups, the proportions of Annexin V-positive $\mathrm{CD} 4{ }^{+}$and $\mathrm{CD} 8{ }^{+} \mathrm{T}$ cells were significantly lower in the recipients of MyD88KO TCDBM than in those of WT TCD-BM (Figure 2g). Expressions of Bax and Caspase 9 by both $T$ cell subsets were also significantly 
reduced in the MyD88KO TCD-BM recipients compared with the latter (Figure 2h). Together, these results suggested that negative regulation of alloreactive CD $45.1^{+} \mathrm{T}$ cells would be also less stringent in the MyD88KO group. As low stringency of negative regulation within the spleens may cause expedited severe intestinal damage by heightening the chances for $\mathrm{T}$ cells to survive and egress out to intestines, we suggest that infiltration of more $\mathrm{T}$ cells into the SIs of MyD88KO group than of WT group on day 13 may also be attributed to the less stringent negative regulation on allo-reactive $\mathrm{T}$ cells.
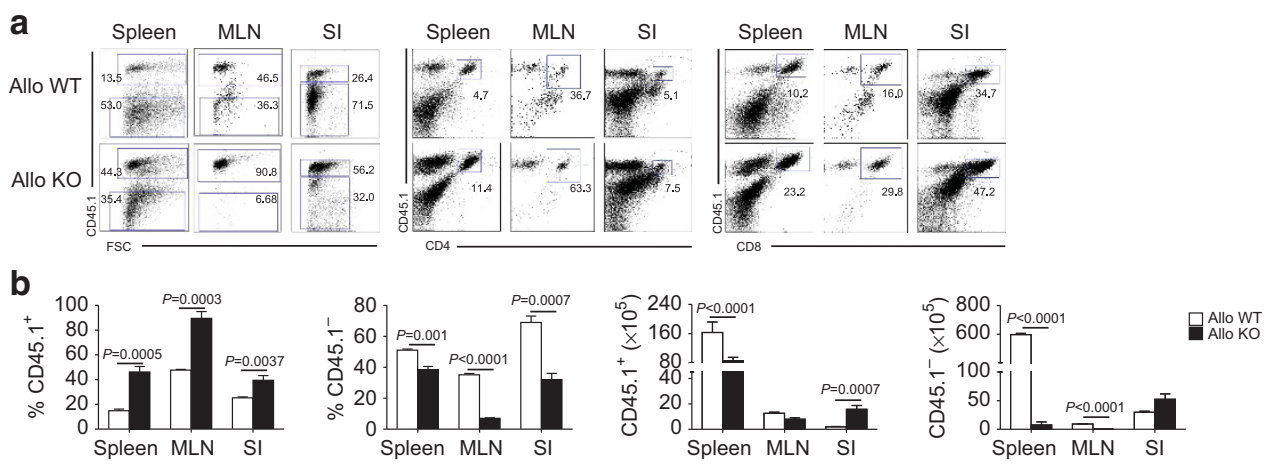

C

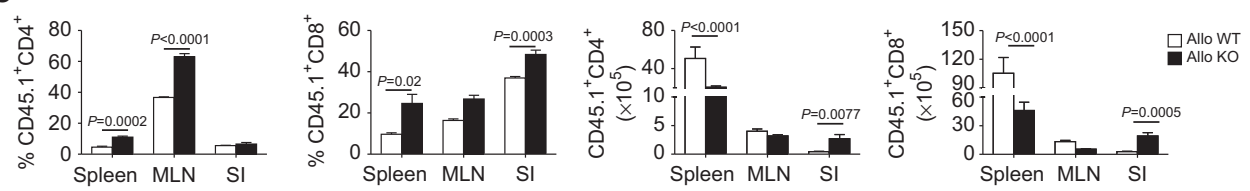

d
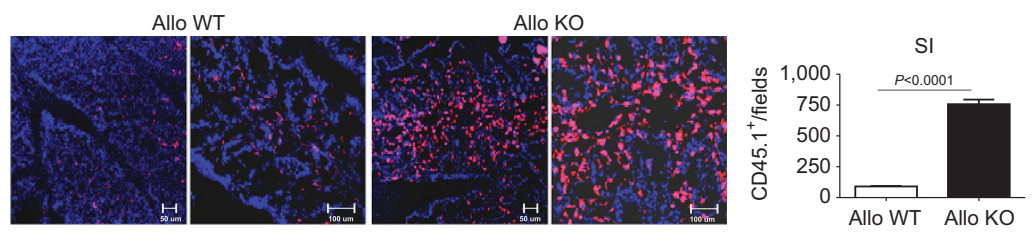
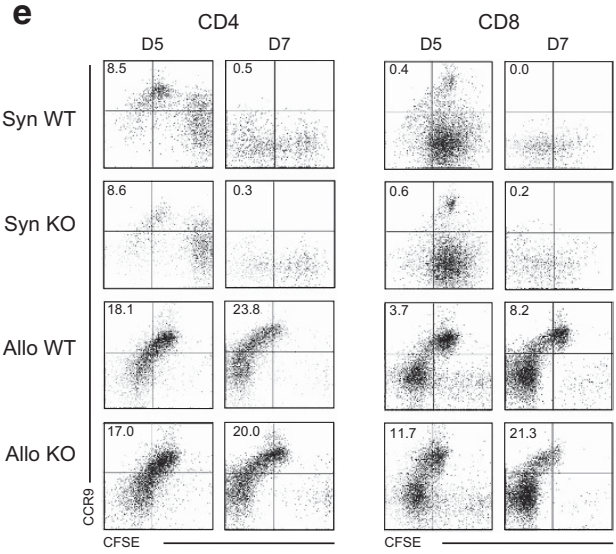

CFSE control

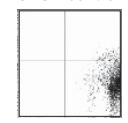

$\mathrm{CD} 45.1^{+} \mathrm{CD} 4^{+}$
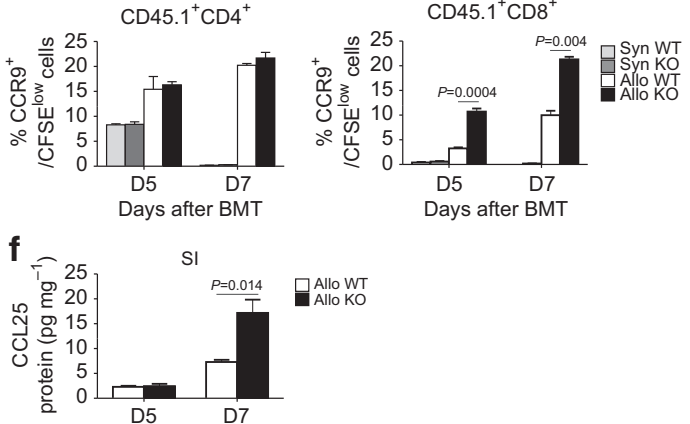

g
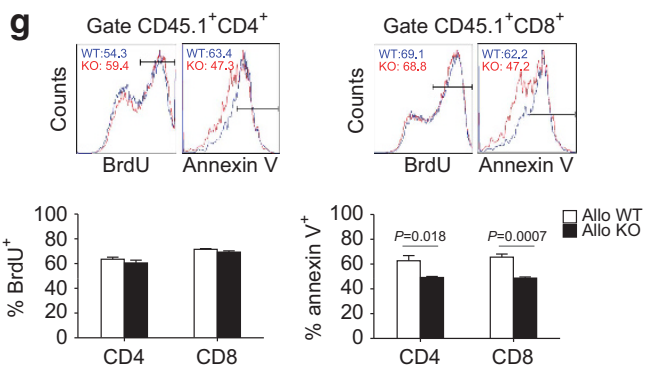

h
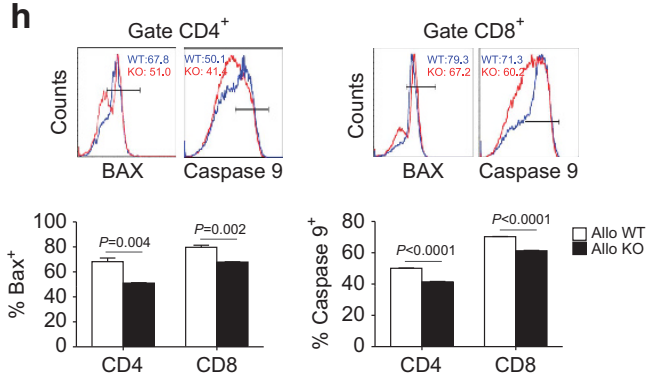

Figure 2 See for caption on page 734 . 


\section{Reduced expansion and ineffective suppressive function of $\mathrm{CD} 11 \mathrm{~b}^{+} \mathrm{Gr}-1^{+}$cells in the allogeneic recipients of MyD88KO TCD-BM}

CD45.1 ${ }^{-}$non-T-cell compartments which were notably decreased in the MyD88KO TCD-BM recipients (Figure 2a) were typed to be $\mathrm{CD} 11 \mathrm{~b}^{+} \mathrm{Gr}-1^{+}$cells (Figure 3a). Especially, the proportions of $\mathrm{Ly} 6 \mathrm{G}^{\text {hi }} \mathrm{Ly} 6 \mathrm{C}^{\text {low }}$ subpopulation within the $\mathrm{CD} 11 \mathrm{~b}^{+} \mathrm{Gr}-1^{+}$cells were significantly lower in the recipients of MyD88KO TCD-BM, compared with those of WT counterparts (Figure 3b). The low proportion of $\mathrm{CD} 11 \mathrm{~b}^{+} \mathrm{Gr}-1^{+}$cells in the MyD88KO group was ascribed to their low-level expansion from transplanted MyD88KO TCD-BM, in response to stimuli produced in the GVHD hosts, because the proportions of $\mathrm{CD} 1 \mathrm{~b}^{+} \mathrm{Gr}-1^{+}$cells in naive BM cells and in peripheral organs of MyD88KO mice (i.e., before transplantation) were comparable to those of WT B6 mice (Supplementary Figure S2b). The granulocytes isolated from MyD88KO mice exhibited myeloperoxidase enzyme activity similar to those from WT mice (Supplementary Figure S2c). $\mathrm{CD}_{11 \mathrm{~b}}{ }^{+} \mathrm{Gr}-1^{+}$cells, which expanded normally in GVHD hosts (recipients of WT TCD-BM), were composed of cells with monocytic and granulocytic morphological features (Figure 3c), consistent with the mixed compositions of Ly6 $6{ }^{\text {low }}$ Ly6 $6 C^{\text {hi }}$ and Ly6G ${ }^{\text {hi }}$ Ly6 $6 C^{\text {low }}$ cells in flow cytometry (Figure 3b); they expressed Arginase-1 (Arg-1) and inducible nitric oxide synthase (iNOS), genes expressed by MDSCs, ${ }^{23}$ at significantly higher levels (upon quantitative RT-PCR (PCR with reverse transcription) analyses) than the $\mathrm{CD} 11 \mathrm{~b}{ }^{+} \mathrm{Gr}-1^{+}$ cells isolated from naïve $\mathrm{B} 6$ mice or from the MyD88KO TCD$\mathrm{BM}$ recipients (Figure 3d). These characteristics identified the $\mathrm{CD}_{11 \mathrm{~b}}{ }^{+} \mathrm{Gr}-1^{+}$cells that expanded in WT GVHD hosts as MDSCs. Consistently, most of the $\mathrm{CD} 11 \mathrm{~b}^{+} \mathrm{Gr}-1^{+}$cells in WT hosts ( $>72 \%$ on average) were F4/80-positive. In comparison, proportions of F4/80-positive cells were significantly lower in the $\mathrm{CD} 11 \mathrm{~b}^{+} \mathrm{Gr}-1^{+}$cells from the spleens and peripheral blood mononuclear cells of the MyD88KO TCD-BM recipients (Figure 3e), which indicated that MDSCs were infrequent within the $\mathrm{CD} 11 \mathrm{~b}^{+} \mathrm{Gr}-1^{+}$cells derived from transplanted
MyD88KO TCD-BM. Therefore, we concluded that incomplete expansion of $\mathrm{CD} 11 \mathrm{~b}^{+} \mathrm{Gr}-1^{+} \mathrm{F} 4 / 80^{+}$MDSCs from MyD88KO TCD-BM increased T-cell infiltration into the intestines, leading to the exacerbation of intestinal GVHD.

We did not detect any meaningful differences in the T-cell production of INF- $\gamma$ by the two groups of TCD-BM recipients (Supplementary Figure S3a). The levels of production of TNF-

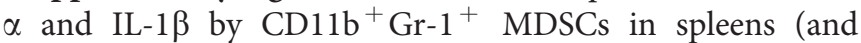
other organs) were also comparable between the two recipients on a per-cell basis (Supplementary Figure S3b). Also, the proportions of Foxp $3^{+} \mathrm{CD} 4{ }^{+}$regulatory T cells in the spleens and SI of the two groups were similar (Supplementary Figure S3c). In the assessment of inflammatory cytokines implicated in pathogenesis of acute GVHD, ${ }^{7}$ differences in serum cytokine levels between the two groups of GVHD hosts were not as dramatic as expected (Supplementary Figure S3d). IL-6 and IL-17 levels were higher and IL-10 level was lower in recipients of MyD88KO TCD-BM than in WT TCD-BM recipients. But the levels of TNF- $\alpha$ and IL- $1 \beta$ were lower in MyD88KO recipients, although the difference in IL-1 $\beta$ levels was not significant. Such findings suggested that variations in cytokine levels might not fundamentally explain the difference in GVHD severity noted between the two groups.

Next, we directly compared the immunosuppressive functions of MDSCs from MyD88KO and WT mice, respectively, by co-incubating $\mathrm{CD}_{11 \mathrm{~b}}{ }^{+} \mathrm{Gr}-1^{+}$cells isolated from the BM of MyD88KO or WT mice with B6-anti-F1 mixed leukocyte cultures. $\mathrm{CD}^{+}{ }^{+}$and $\mathrm{CD} 8{ }^{+}$T cells co-incubated with CD11b ${ }^{+}$ Gr- ${ }^{+}$cells from MyD88KO mice exhibited more extensive CFSE-dilution and less Annexin V-staining than those coincubated with the $\mathrm{CD}_{11 \mathrm{~b}}{ }^{+} \mathrm{Gr}-1^{+}$cells from WT mice (Figure 3f,g). The suppressive function exerted by MDSCs depended on the relative levels of such cells compared with $\mathrm{T}$ cells, in agreement with a previous report of the ratio-dependent MDSC function. ${ }^{24}$ The same results were obtained when the suppression of CD3/CD28-stimulated CD4 ${ }^{+}$and CD8 ${ }^{+}$T cells were used as a readout of MDSC function (Supplementary Figure S3e,f, respectively). In addition, when $\mathrm{CD} 11 \mathrm{~b}^{+} \mathrm{Gr}-1^{+}$

Figure 2 Effect of MyD88 deficiency in TCD-BM on the expansion and infiltration of donor-type T cells into the intestine. Lethally irradiated F1 recipients were given $5 \times 10^{6}$ WT or MyD88KO TCD-BM cells plus $1 \times 10^{6}$ purified WT T cells isolated from congenic B6 donors (CD45. $1^{+} ; n=5$, each group, allo WT and allo KO). (a) The expansion of donor T cells $\left(\mathrm{CD} 45.1^{+}\right)$and non-T cell (CD45.1 ${ }^{-}$) compartments in the spleen, mesenteric lymph node (MLN) and small intestines (SI) were assessed by staining of the leukocytes for expressions of CD45.1, CD4, and CD8 on day 13 post transplantation. (b) The frequencies CD45.1 ${ }^{+}$cells (donor-type T cells) and CD45.1- cells (non-T-cell compartment) in the leukocytes isolated from different organs shown in a and their absolute numbers are plotted. (c) The frequencies and numbers of donor-type $\left(\mathrm{CD} 45.1^{+}\right) \mathrm{CD} 4^{+}$and CD8 ${ }^{+} \mathrm{T}$ cells are plotted. Data are presented as means \pm s.e.m. and are representative of duplicate experiments. (d) Infiltration of CD $45.1^{+}$cells in the SI during GVHD. SI were harvested from F1 recipient mice $(n=5)$ on day 13 and sectioned for staining with anti-CD45.1 Ab as described in "Methods". Magnification: $\times 100$ (left) and $\times 200$ (right). (e) Lethally irradiated B6 (syn WT and syn KO) or F1 (allo WT and allo KO) recipients underwent transplantation from WT B6. Ly-5a (CD45.1) donor T cells plus either WT or MyD88KO TCD-BM cells. Splenocytes from each group of mice were isolated and analyzed on days 5 and 7 ( $n=5$ per group). CFSE-labeled T cells before the transplantation were used as CFSE-positive controls. Expression of CCR9 was examined by gating the donor T-cellderived $\mathrm{CD} 45.1^{+} \mathrm{CD} 4{ }^{+}$or $\mathrm{CD} 45.1^{+} \mathrm{CD}^{+}$cells. Data from one of five independent experiments are shown. FACS data of syngeneic controls are shown. Data are presented as means \pm s.e.m. (f) Quantitative analysis of CCL25 protein production was determined by enzyme-linked immunosorbent assay on days 5 and 7. CCL25 expressions of SI were compared between the WT and MyD88KO recipients $(n=5$ per group). Data from one of two replicate experiments that yielded similar results are shown. Data are presented as means \pm s.e.m. (g, h) After irradiation, F1 recipients underwent transplantation with WT B6.Ly-5a (CD45.1) donor T cells plus either WT or MyD88KO TCD-BM cells. Splenocytes were isolated and analyzed on day 4. Cells are gated on either CD45.1 ${ }^{+} \mathrm{CD} 4{ }^{+}$or $\mathrm{CD} 45.1{ }^{+} \mathrm{CD} 8{ }^{+}$cells, and the percentages of BrdU- and Annexin V-positive cells (g), and Bax-and Caspase 9 -positive cells $(\mathbf{h})$ were determined ( $n=6$ per group). Data in $\mathbf{g}$ and $\mathbf{h}$ from one of two replicate experiments that yielded similar results are shown. Data are presented as means \pm s.e.m. BMT, bone marrow transplantation; CFSE, carboxyfluorescein succinimidyl ester; GVHD, graft-vs.-host disease; KO, knockout; TCD-BM, T-cell depleted bone marrow; WT, wild type. 
a

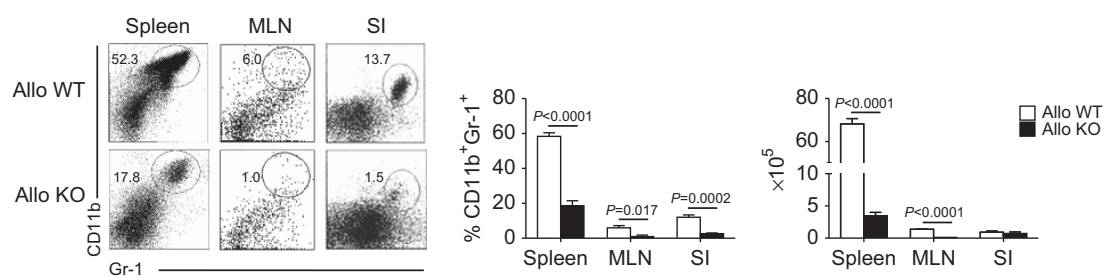

b
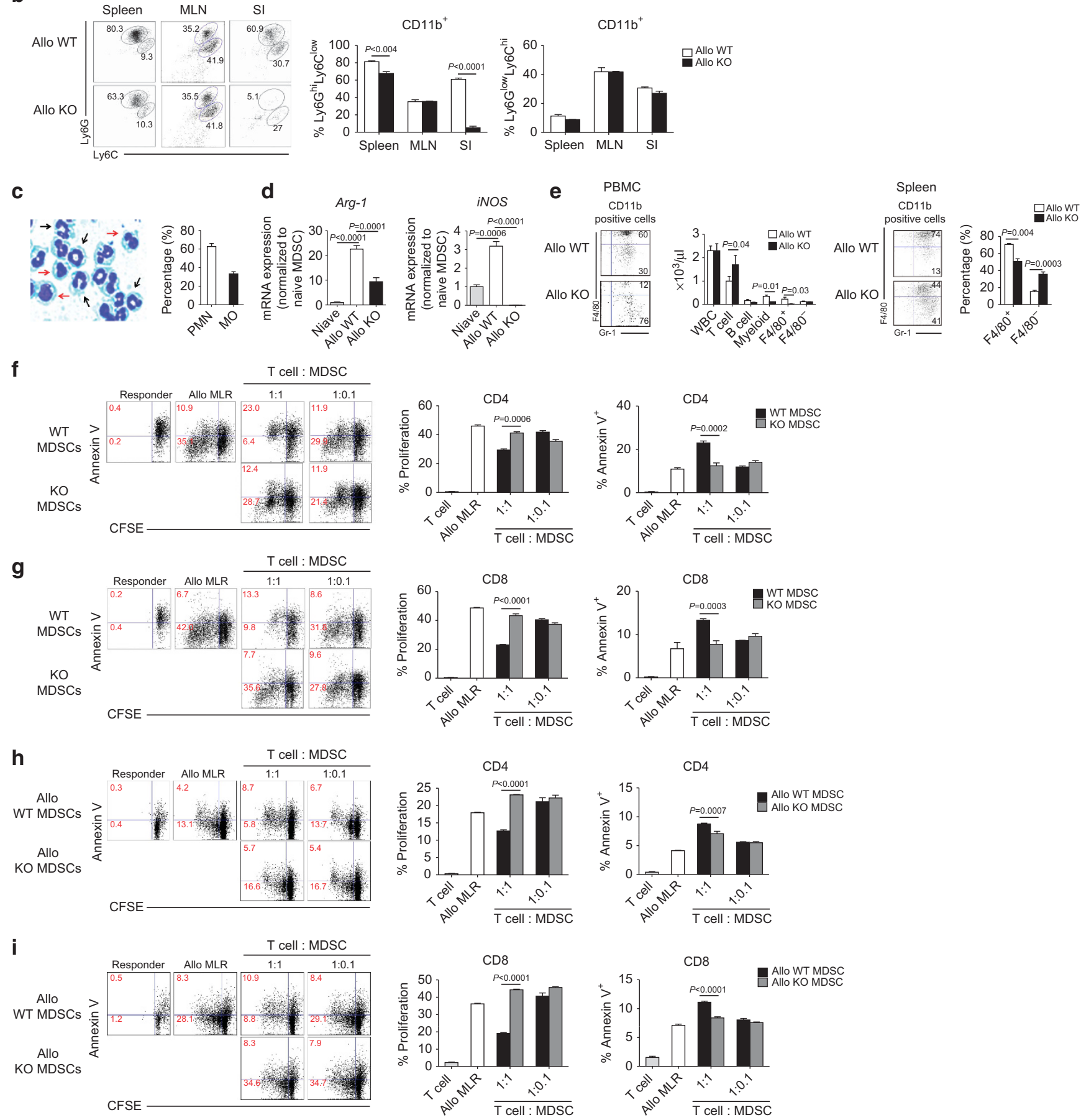

Figure 3 See for caption on page 736 . 
cells isolated from the BM of GVHD hosts that had been transplanted with either WT or MyD88KO TCD-BM were tested for their suppressive activity, those from the recipients of MyD88KO TCD-BM exhibited a reduced suppressive function, compared with their WT counterparts (Figure $\mathbf{3 h}, \mathbf{i}$ ), which is probably related to the decreased expression of Arg-1 and iNOS genes by the cells of MyD88KO TCD-BM origin (Figure 3d). Thus, GVHD exacerbation upon transplantation of MyD88KO TCD-BM was associated not only with inadequate expansion of MyD88KO-MDSCs in GVHD hosts, but also with the ineffective negative regulation of alloreactive donor $\mathrm{T}$ cells.

\section{Increased mortality and insufficient MDSC expansion are evident even in miHA-mismatched recipients of MyD88KO TCD-BM}

To determine whether the increase in GVHD mortality and the inadequate MDSC expansion noted after allogeneic transplantation of MyD88KO TCD-BM could be reproduced in other GVHD models, we induced acute GVHD in irradiated BALB.B mice used as recipients of WT B6 T cells and MyD88KO or WT B6 TCD-BMs; this is an miHA-mismatched GVHD model. ${ }^{25}$ We next performed flow cytometric analyses as described above. Also, in this model, GVHD severity and mortality were exacerbated in recipients of MyD88KO TCDBM. These animals had a shorter median survival time (10 days) and more serious disease symptoms than did recipients of WT TCD-BM (median survival time: 24 days; Supplementary Figure S4a-c). The numbers of $\mathrm{CD}^{+}$and $\mathrm{CD} 8{ }^{+} \mathrm{T}$ cells infiltrating the intestines were also higher in recipients of MyD88KO TCD-BM than WT TCD-BM, and expansion of $\mathrm{CD} 11 \mathrm{~b}{ }^{+} \mathrm{Gr}-1^{+}$MDSCs was significantly reduced in recipients of MyD88KO TCD-BM (Supplementary Figure S4d) on day 7 post transplantation. All CD4 ${ }^{+}$and $\mathrm{CD} 8^{+} \mathrm{T}$, and $\mathrm{CD} 11 \mathrm{~b}^{+}$ and $\mathrm{Gr}-1^{+}$cells were of donor origin after day 7 , as shown by typing based upon allelic polymorphisms at the $\beta 2 \mathrm{~m}$ locus (B6: $\beta 2 \mathrm{~m}^{\mathrm{b}}$; Supplementary Figure $\left.\mathbf{S} 4 \mathbf{e}, \mathbf{f}\right)$. Together, these data confirm that transplantation with MyD88KO TCD-BM during the induction of GVHD aggravated the disease, and was associated with a high-level $\mathrm{T}$-cell infiltration into the intestine and insignificant expansion of MDSCs, even in miHAmismatched GVHD hosts.

\section{Supplementation of recipients of MyD88KO TCD-BM with WT-MDSCs attenuates GVHD severity and reduces donor T-cell infiltration into the intestines}

Next, we explored whether supplementation of GVHD hosts with WT-MDSCs during the course of GVHD could compensate the lack of MDSC function and alleviate GVHD severity in MyD88KO TCD-BM recipients. To this end,

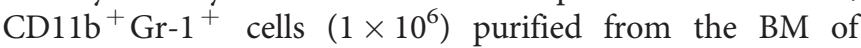
WT or MyD88KO mice were injected into GVHD hosts that had received MyD88KO TCD-BM on days 3, 5, and 7 post transplantation (MDSCs from the two different donors were similarly homogeneous in nature, exhibiting features of granulocyte-monocyte precursors; Figure 4a). As expected, supplementation with WT-CD $11 \mathrm{~b}^{+} \mathrm{Gr}-1^{+}$cells attenuated GVHD severity in the recipients of MyD88KO TCD-BM. The disease severity was similar to or less than that noted in another control GVHD group that had received WT TCD-BM, without any further supplementation (Figure $4 \mathbf{b}$ ). In contrast, the attenuating effect evident after supplementation with WT$\mathrm{CD}_{11 \mathrm{~b}}{ }^{+} \mathrm{Gr}-1^{+}$cells was not observed upon supplementation with MyD88KO-CD11b ${ }^{+}$Gr- $1^{+}$cells, and this difference was emphasized when intestinal pathology was compared (Figure 4c). In addition, supplementation with half dose of

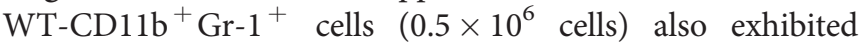
attenuating effects (Supplementary Figure S5) but less than observed after full-dose treatment, implying that the dose of $\mathrm{CD} 1 \mathrm{~b}^{+} \mathrm{Gr}-1^{+}$cell (or abundance) influenced the degree of GVHD protection. The profiles of leukocyte infiltration into the spleen, MLN and intestine were reversed upon supplementation of $\mathrm{WT}-\mathrm{CD} 11 \mathrm{~b}^{+} \mathrm{Gr}-1^{+}$

Figure 3 Effect of MyD88-deficiency in TCD-BM on the expansion, and in vivo and in vitro suppressive function of CD11b ${ }^{+}$Gr-1 $1^{+}$MDSCs. Lethally irradiated F1 recipients were given $5 \times 10^{6}$ WT or MyD88KO TCD-BM cells plus $1 \times 10^{6}$ purified WT T cells isolated from WT B6 donors $(n=5$, each group, allo WT and allo KO). (a, b) On day 13 thereafter, the spleen, mesenteric lymph node (MLN) and small intestine (SI) were harvested. (a) Expression levels of $\mathrm{CD} 11 \mathrm{~b}^{+} \mathrm{Gr}-1^{+}$were measured via flow cytometric analysis. The proportions and absolute numbers of CD $11 \mathrm{~b}^{+} \mathrm{Gr}-1^{+}$cells are shown. Data are presented as means \pm s.e.m. and are representative of duplicate experiments. (b) Proportions of Ly6G ${ }^{\text {hi }} L y 6 C^{\text {low }}$ and Ly6G ${ }^{\text {low }}$ Ly6C ${ }^{\text {hi }}$ populations in the gated $\mathrm{CD} 11 \mathrm{~b}^{+}$cells are shown. Data are presented as means \pm s.e.m. and are representative of duplicate experiments. $(\mathbf{c}) \mathrm{CD} 11 \mathrm{~b}^{+} \mathrm{Gr}-1^{+}$cells were isolated from the spleens of allogeneic recipients transplanted with WT TCD-BM and T cells and were processed for May-Grünwald and Giemsa-staining after cytospin preparation $(n=3)$. The percentages of cells in isolated populations exhibiting monocytic (MO; indicated as red arrowhead) and polymorphonuclear (PMN; indicated as black arrowhead) features are shown. (d) CD11b ${ }^{+} \mathrm{Gr}-1^{+}$cells isolated from naive B6 mice and allogeneic recipients of T cells plus WT or MyD88KO TCD-BM ( $n=3$, naive, allo WT and allo KO) were compared in terms of the expression of $\operatorname{Arginase-1}$ (Arg-1) and inducible nitric oxide synthase (iNOS) via quantitative RT-PCR. (e) Peripheral blood mononuclear cells (PBMCs) were isolated from GVHD hosts transplanted with WT or MyD88KO TCD-BM (allo WT and allo KO, respectively) on day 13 post transplantation ( $n=3$ per group), and analyzed in terms of the counts of $\mathrm{T}\left(\mathrm{CD}^{+}\right), \mathrm{B}\left(\mathrm{B} 22 \mathrm{O}^{+}\right)$, myeloid $\left(\mathrm{CD} 11 \mathrm{~b}^{+}\right)$cells of $\mathrm{H}-2^{\mathrm{d}}$-negative donor origin. $\mathrm{F} 4 / 80$-staining was included in the analysis of $\mathrm{CD} 11 \mathrm{~b}^{+} \mathrm{Gr}-1^{+}$ cells in PBMCs and spleens. Representative flow cytometry dot plots of frequencies and the corresponding cell numbers are shown. Data are presented as means \pm s.e.m. The data shown in a-d are representative of two independent experiments. (f, $\mathbf{g})$ Purified T cells $\left(2 \times 10^{5}\right)$ were cultured with irradiated F1 splenocytes $\left(2 \times 10^{5}\right)$ in the presence of purified WT or MyD88KO CD11b ${ }^{+} \mathrm{Gr}-1^{+}$cells (WT MDSCs or KO MDSCs, $2 \times 10^{5}$ or $0.2 \times 10^{5}$ ). The percentages of CFSE and Annexin V on the CD4 ${ }^{+}(\mathbf{f})$ and $C D 8^{+}(\mathbf{g})$ T cells were determined after 4 days of co-culture $(n=3)$. (h, i) Suppression assays were performed according to the same procedure as described in $\mathbf{f}$ and $\mathbf{g}$ above, using CD $11 \mathrm{~b}^{+} \mathrm{Gr}-1^{+}$cells $\left(2\right.$ or $\left.0.2 \times 10^{5}\right)$ purified from GVHD recipients transplanted with $5 \times 10^{6}$ WT (allo WT MDSCs) or MyD88KO TCD-BM cells (allo KO MDSCs) on day 13 post transplantation $(n=5)$. The percentages of CFSE and Annexin V-positive $\mathrm{CD}^{+}{ }^{+}$(h) and $\mathrm{CD}^{+}{ }^{+}$(i) T cells were determined. All of the data are presented as means \pm s.e.m. Data in $\mathbf{f}-\mathbf{i}$ represent two independent experiments that yielded similar results. BMT, bone marrow transplantation; CFSE, carboxyfluorescein succinimidyl ester; GVHD, graft-vs.host disease; KO, knockout; MDSC, myeloid-derived suppressor cell; TCD-BM, T-cell depleted bone marrow; WT, wild type. 
cells. Thus, $\mathrm{CD}^{+}{ }^{+}$and $\mathrm{CD} 8{ }^{+} \mathrm{T}$-cell numbers were reduced in the intestines of recipients of MyD88KO TCD-BM upon supplementation with WT-MDSCs, compared with animals supplemented with MyD88KO-CD11 ${ }^{+} \mathrm{Gr}-1^{+}$cells (Figure $4 \mathbf{d}-\mathbf{g}$ ). The numbers of $\mathrm{CD} 11 \mathrm{~b}^{+} \mathrm{Gr}-1^{+}$cells also increased in all the tested organs after WT-cell supplementation (Figure 4h). Together, the results showed that $\mathrm{CD} 11 \mathrm{~b}^{+} \mathrm{Gr}-1^{+}$cells from MyD88KO mice were defective in terms of expansion and exertion of a protective role in vivo in GVHD hosts. a

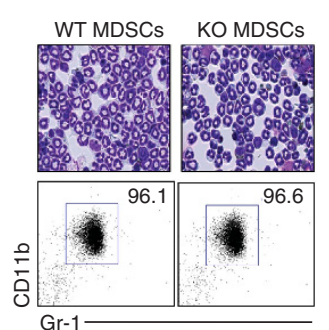

b

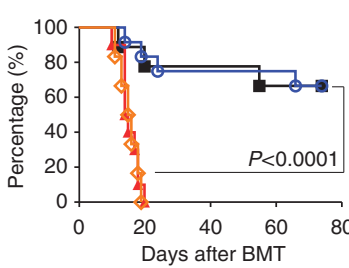

GVHD score

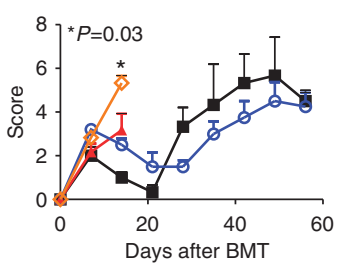

Body weight loss

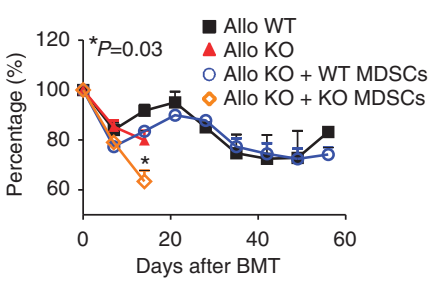

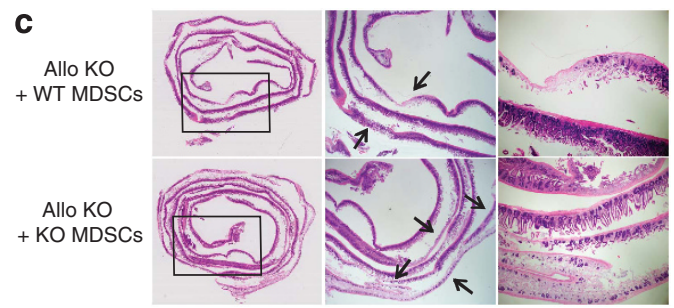

C

e

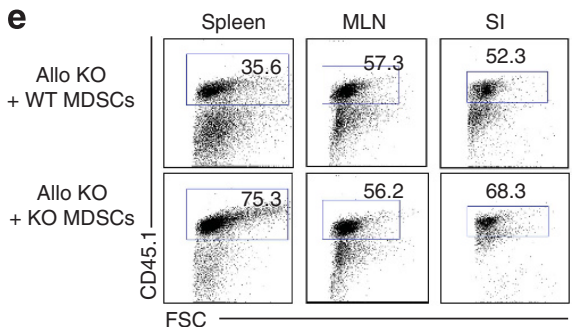

f

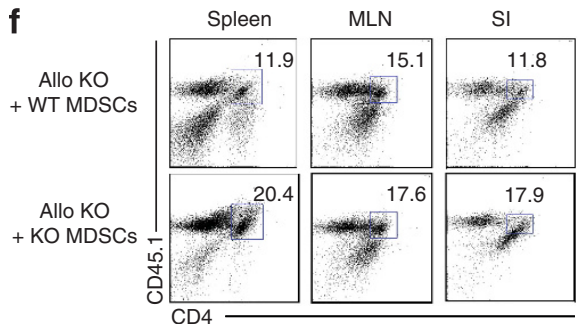

g

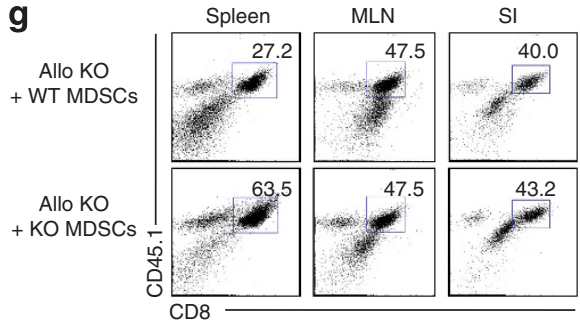

h

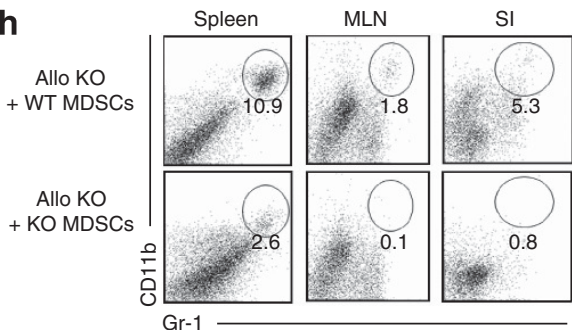

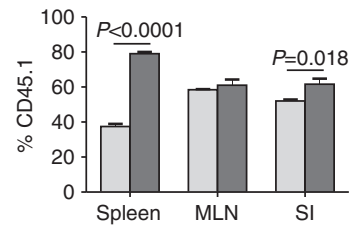

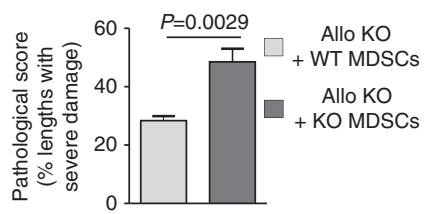

d Total cell count
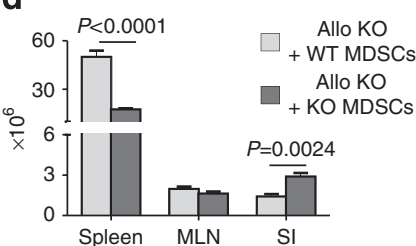
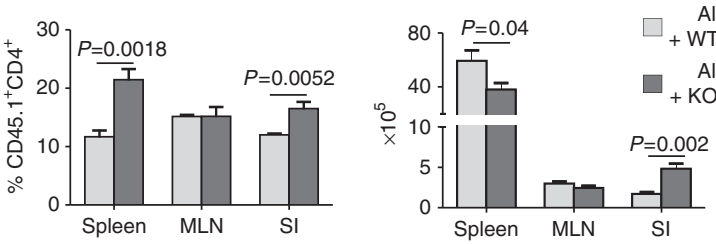
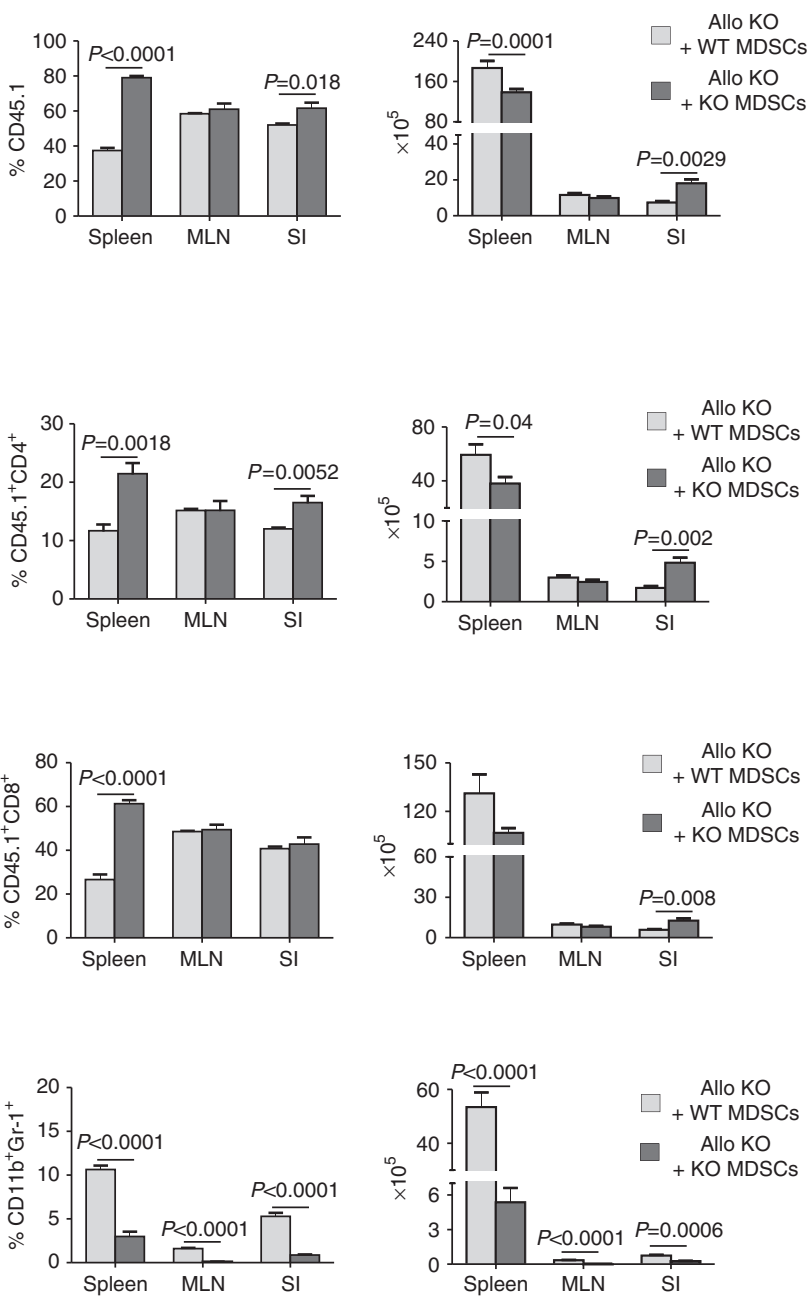

Figure 4 See for caption on page 738 . 


\section{Recipients of TCD-BM from WT donor mice injected with LPS develop less severe GVHD and show reduced levels of intestinal T-cell recruitment}

To verify the importance of MyD88-mediated signaling in $\mathrm{CD} 1 \mathrm{~b}^{+} \mathrm{Gr}-1^{+}$cells in reducing the severity of GVHD, we examined whether transplantation with TCD-BM cells contain-

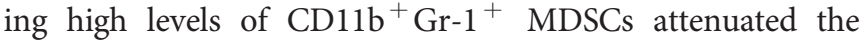
severity of GVHD. As repeated injection of LPS elicited the development of $\mathrm{CD}_{11 \mathrm{~b}}{ }^{+} \mathrm{Gr}-1^{+} \mathrm{MDSCs}^{26}$ we injected BM donors with LPS before the preparation of TCD-BM, and confirmed that pretreatment with LPS increased CD11b Gr- $1^{+}$cell frequencies (Figure 5a) as well as the levels of $M y d 88$ transcripts (Figure $5 \mathbf{b}$ ) in the TCD-BM inoculum. Transplantation with TCD-BM isolated from the LPSpretreated donors significantly improved GVHD survival and attenuated disease severity, compared with that after transplantation with control TCD-BM that had been pretreated with the vehicle (Figure 5c). This GVHD alleviation by LPSpretreated TCD-BM was also observed in the miHAmismatched GVHD model (Figure 5d). The pattern of organ infiltration by CD45.1 ${ }^{+} \mathrm{T}$ cells differed in the two groups; the extent of intestinal T-cell infiltration was lower (Figure 5e-h), and the proportions of $\mathrm{CD} 11 \mathrm{~b}^{+} \mathrm{Gr}-1^{+}$cells in target organs were higher (Figure 5i), in the test recipients than in controls. Therefore, elevated $\mathrm{CD}_{11 \mathrm{~b}}{ }^{+} \mathrm{Gr}-1^{+}$cell levels in TCD-BM transplants, achieved via MyD88-signaling triggered by LPS-pretreatment, ameliorated fatal intestinal GVHD.

\section{The ratio of $\mathrm{T}$ cells/MDSCs at engraftment predicts the development of intestinal GVHD}

$\mathrm{CD}_{11 \mathrm{~b}}{ }^{+} \mathrm{Gr}-1^{+}$MDSCs have a direct relevance to the control of GVHD development. ${ }^{18}$ Retrospectively, we found that higher proportions of T cells over CD $11 \mathrm{~b}^{+} \mathrm{Gr}-1^{+}$MDSCs in peripheral blood or spleens (high $\mathrm{T}$ cell/MDSC ratios) positively correlated with the severity of intestinal GVHD in the several contexts of the present study (Figure 6a-c). We, therefore, wondered whether MDSC expansion levels could be used to predict intestinal GVHD severity. We calculated T cell/ MDSC ratios in peripheral blood mononuclear cells of the consecutively transplanted patients (Supplementary Table 1) at the time of engraftment. We determined the full engraftment when the host cells showed almost $100 \%$ donor-cell chimerism. Human MDSCs express lineage-HLA-DR ${ }^{-} \mathrm{CD} 11 \mathrm{~b}^{+} \mathrm{CD} 33^{+}$ phenotypes. $^{27}$ The calculated ratios of $\mathrm{T}$ cell/MDSCs were significantly higher in patients with intestinal GVHD $\geqslant$ grade 2 (Figure 6d) than in others, suggesting that this parameter (T/MDSC ratio at engraftment) may be used to predict intestinal GVHD severity. The results of univariate and multivariate analysis regarding occurrence of acute intestinal GVHD are shown (Supplementary Table 2). However, this parameter was found not to be significantly associated with the occurrence of overall acute GVHD $\geqslant$ grade 2 (data not shown), demonstrating that the development of intestinal GVHD was particularly associated with inadequate expansion of MDSCs immediately after clinical allo-HSCT.

\section{DISCUSSION}

In the present study in which we used two murine models, we found that expression of MyD88 in a TCD-BM transplant was important for protection against fatal acute GVHD of the intestine. Lack of MyD88 expression in the TCD-BM transplant caused incomplete expansion and functional expression of $\mathrm{CD}_{11 \mathrm{~b}}{ }^{+} \mathrm{Gr}-1^{+}$MDSCs in recipients, and an increase in MDSC levels in the TCD-BM (achieved via pretreatment of donors with LPS) alleviated GVHD severity and infiltration of $\mathrm{T}$ cells into the intestine. Clinically, relatively high T/MDSC ratios in blood correlated with the severity of intestinal GVHD in human patients.

Donor-derived BM cells repopulate the hematopoietic systems of recipients, but may also participate in GVHD pathogenesis, in that donor APCs present host antigens to alloreactive donor $\mathrm{T}$ cells and exacerbate GVHD. ${ }^{28}$ However, such roles for donor-derived immune cells, other than donor APCs and T cells, in GVHD pathogenesis, have rarely been reported. We found that lack of MyD88 expression in donor TCD-BM was detrimental in terms of GVHD survival, despite the absence of MyD88-mediated activation of donor APCs. In a GVHD model using a BM chimera as a host, in which hematopoietic cells were replaced by MyD88KO BM-derived

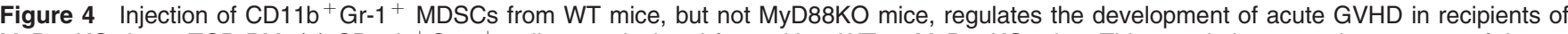
MyD88KO donor TCD-BM. (a) CD11 b ${ }^{+} \mathrm{Gr}-1^{+}$cells were isolated from either WT or MyD88KO mice. This population comprises $\geqslant 95 \%$ of the $\mathrm{CD} 11 \mathrm{~b}^{+} \mathrm{Gr}-1^{+}$MDSCs fraction by flow cytometric analysis. Each May-Grünwald and Giemsa-stained cytospin preparation is shown. (b) Lethally irradiated F1 recipients were given $5 \times 10^{6}$ MyD88KO TCD-BM cells plus $1 \times 10^{6}$ purified WT T cells from allogeneic B6 donors. Mice exhibiting GVHD that had received MyD88KO TCD-BM cells were injected with $1.0 \times 10^{6} \mathrm{CD} 11 \mathrm{~b}^{+} \mathrm{Gr}-1^{+}$MDSCs isolated from WT or MyD88KO mice $(n=12$; allo $\mathrm{KO}+$ WT MDSCs or allo KO + KO MDSCs, respectively) at days 3, 5, and 7 post transplantation. In parallel, GVHD was induced with WT or MyD88KO TCD-BM plus WT T cells, but without MDSC supplementation ( $n=9$; allo WT or allo KO, respectively). Data from two similar experiments are combined. The Kaplan-Meier survival curves are shown (allo KO + WT MDSCs vs. allo KO + KO MDSCs by the Wilcoxon rank-sum test). The data are presented as means \pm s.e.m. (c) Small bowels were collected for histological examination on day 13 after transplantation. Pathological scores were compared between mice receiving WT MDSCs and MyD88KO MDSCs, as shown in Figure 1d. Representative pathological findings of severe lesions are indicated as arrows. The middle figures $(\times 12.5)$ are the areas within the black squares, and the right figures $(\times 200$ images $)$ are representative of regions with severe damage. Data are presented as means \pm s.e.m. (d) The absolute numbers of total cells in the spleens, mesenteric lymph nodes (MLN) and small intestine (SI) were assessed from the allogeneic recipients of $1.0 \times 10^{6} \mathrm{CD} 11 \mathrm{~b}^{+} \mathrm{Gr}-1^{+}$MDSCs on day 13 post transplantation. (e-g) The frequencies and absolute numbers of donor T cells (e) with donor-type $\mathrm{CD}^{+}(\mathbf{f})$ and $\mathrm{CD}^{+}(\mathbf{g}) \mathrm{T}$ cells analyzed via flow cytometry are shown. (h) Expression of $\mathrm{CD} 11 \mathrm{~b}^{+} \mathrm{Gr}-1^{+}$was measured using FACS analysis. The frequencies and absolute numbers of $\mathrm{CD} 11 \mathrm{~b}^{+} \mathrm{Gr}-1^{+}$cells are shown. Data are presented as means \pm s.e.m. Data in $\mathbf{c}-\mathbf{h}$ are representative of duplicate experiments ( $n=6$ per experiment). BMT, bone marrow transplantation; FACS, fluorescenceactivated cell sorting; GVHD, graft-vs.-host disease; KO, knockout; MDSC, myeloid-derived suppressor cell; TCD-BM, T-cell depleted bone marrow; WT, wild type.
} 
a
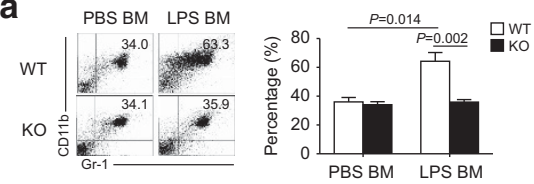

C

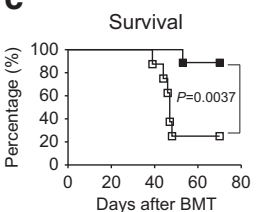

d

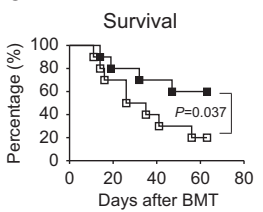

$\mathrm{B} 6 \Rightarrow \mathrm{B} 6 \mathrm{D} 2 \mathrm{~F} 1$

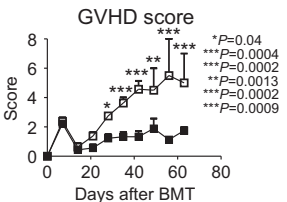

B6 $\Rightarrow$ BALB.B

GVHD score

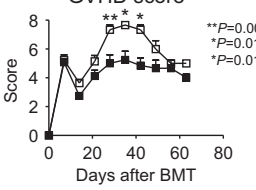

b
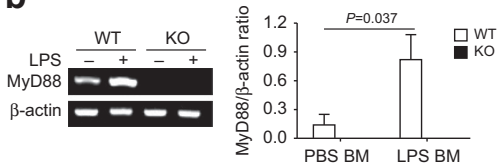

Body weight loss

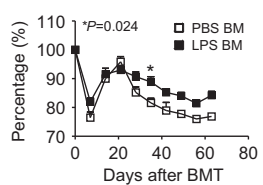

e

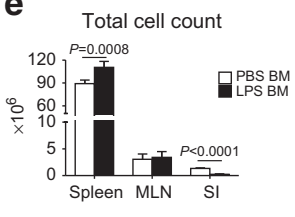

f
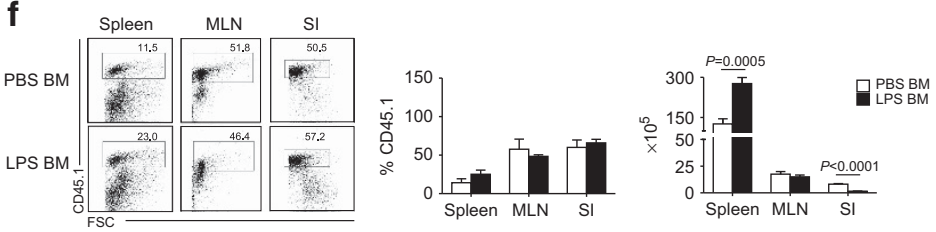

g
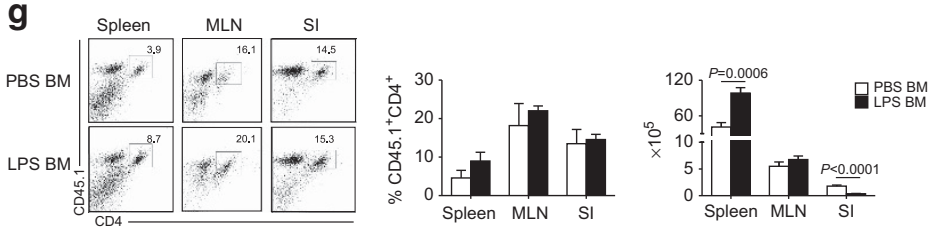

h
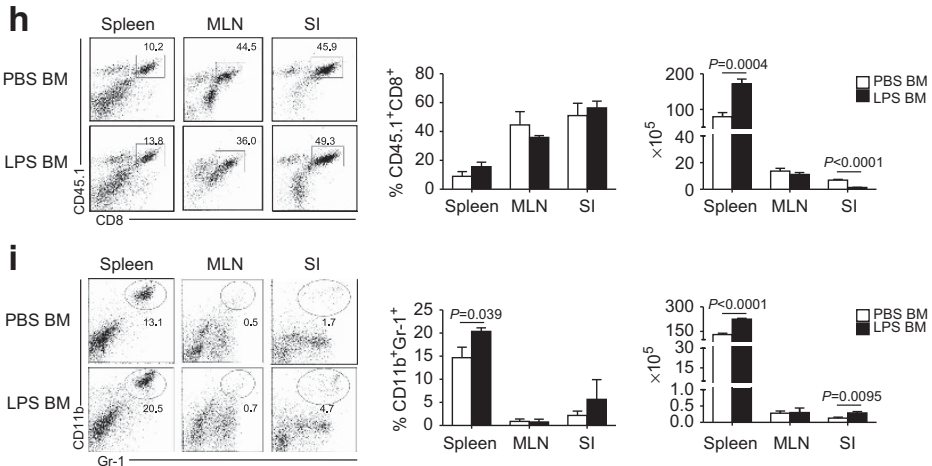

Figure 5 Injection of a sublethal dose of LPS into donor mice increases the frequency of CD11 $\mathrm{b}^{+} \mathrm{Gr}-1^{+}$cells, with elevation of the MyD88 expression level in the TCD-BM, and attenuates the severity of acute GVHD. WT or MyD88KO B6 donor mice were subjected to intraperitoneal (i.p.) injection of LPS at $2 \mathrm{mg} \mathrm{kg}^{-1}$ for 3 days (LPS BM). Mice not receiving LPS received an equal amount of the LPS vehicle (PBS i.p., PBS BM). (a) TCD-BM CD11b ${ }^{+}$Gr- $1^{+}$ cells were examined by flow cytometry for each group $(n=5)$. Data are presented as means \pm s.e.m. (b) MyD88 mRNA expression in TCD-BM was compared between mice injected with LPS and vehicle $(n=3)$. (c) Lethally irradiated F1 recipients were given $5 \times 10^{6}$ LPS- or PBS-treated donor TCDBM cells plus $2 \times 10^{6}$ purified WT T cells from allogeneic B6 donors ( $n=9 / \mathrm{LPS} \mathrm{BM}, n=8 / \mathrm{PBS}$ BM). Data from two similar experiments are combined. Kaplan-Meier survival curves are shown (LPS BM vs. PBS BM by the Wilcoxon rank-sum test). Data are presented as means \pm s.e.m. (d) Alleviation of GVHD by transplantation with LPS-pretreated TCD-BM in a miHA-mismatched allo-HSCT model. BALB.B mice were transplanted with LPS- or PBSpretreated TCD BM cells $\left(5 \times 10^{6}\right)$ plus $5 \times 10^{6}$ purified T cells from allogeneic B6 mice after total body irradiation $(n=10$ in each group, LPS BM and PBS BM). The corresponding Kaplan-Meier survival curves are shown (LPS BM vs. PBS BM by the Wilcoxon rank-sum test). The clinical severity of acute GVHD was assessed weekly. Data shown represent two independent experiments. (e-i) Lethally irradiated F1 recipients were given $5 \times 10^{6}$ LPS- or PBS-treated donor TCD-BM cells plus $1 \times 10^{6}$ purified WT T cells from allogeneic B6 donors ( $n=6$ per group). (e) The absolute numbers of total cells in the spleens, mesenteric lymph nodes (MLN) and small intestine (SI) were assessed on day 13 post transplantation. (f-h) The frequencies and absolute numbers of donor T cells (f), and donor-type $\mathrm{CD}^{+}(\mathbf{g})$ and $\mathrm{CD}^{+}(\mathbf{h}) \mathrm{T}$ cells analyzed via flow cytometry are shown. (i) The expression levels of $\mathrm{CD} 11 \mathrm{~b}^{+} \mathrm{Gr}-1^{+}$were measured via FACS. The frequencies and absolute numbers of CD11 $\mathrm{b}^{+} \mathrm{Gr}-1^{+}$cells are shown. The data are presented as means \pm s.e.m. Data in $\mathbf{a}, \mathbf{b}$ and $\mathbf{e}-\mathbf{i}$ are representative of duplicate experiments. BMT, bone marrow transplantation; FACS, fluorescence-activated cell sorting; GVHD, graft-vs.-host disease; KO, knockout; LPS, lipopolysaccharide; PBS, phosphate-buffered saline; TCD-BM, T-cell depleted bone marrow; WT, wild type. 
a

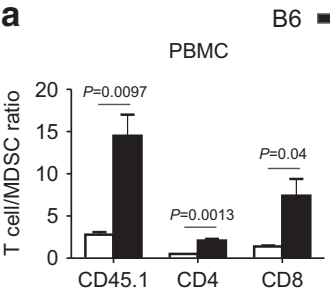

$\mathrm{B} 6 \mathrm{~B} 6 \mathrm{D} 2 \mathrm{~F} 1$

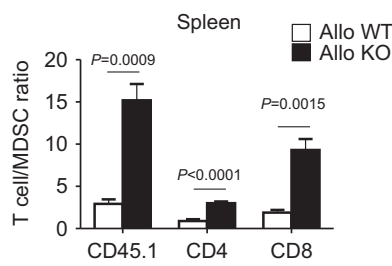

b

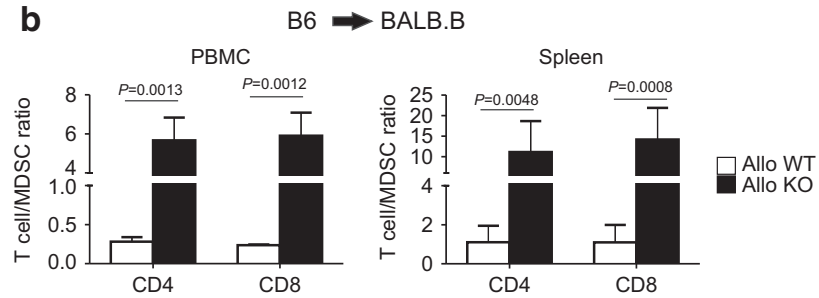

C $\mathrm{B} 6 \Rightarrow \mathrm{B} 6 \mathrm{D} 2 \mathrm{~F} 1+\mathrm{MDSCs}$
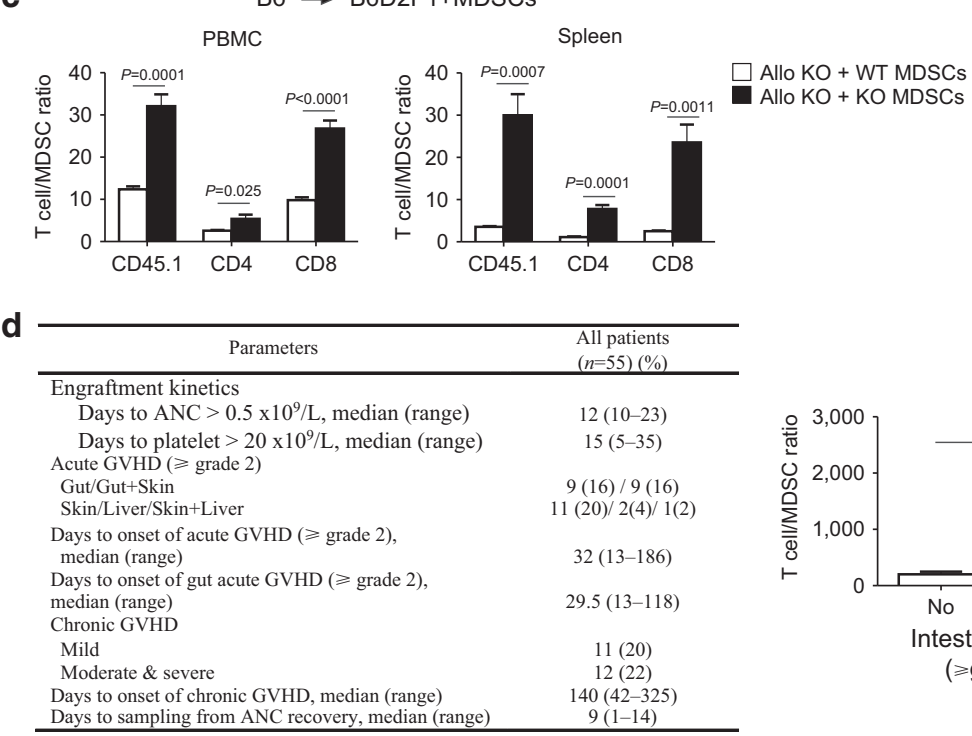

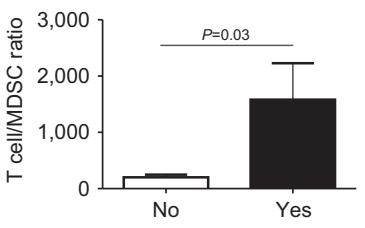

Intestinal GVHD

( $\geqslant$ grade 2)

Figure 6 T cell/MDSC ratio at engraftment predicts the development of intestinal GVHD. (a) The ratios of T cells/CD11b ${ }^{+} \mathrm{Gr}-1^{+}$cells at day 13 in the peripheral blood mononuclear cells (PBMCs) and the spleen were determined in the B6 $\rightarrow$ F1 GVHD model. Individual values from the two independent experiments described in Figure 1 were considered and the data are presented as means \pm s.e.m. (b) The ratios of T cells/CD $11 b^{+}$Gr-1 ${ }^{+}$cells at day 7 in PBMCs and the spleen were determined in the B6 $\rightarrow$ BALB.B GVHD model. The four independent experimental data sets described in Supplementary Figure S4 are considered and data are presented as means \pm s.e.m. (c) The ratios of T cells/CD $11 b^{+} \mathrm{Gr}-1^{+}$cells at day 13 in PBMCs and the spleen were determined in the B6 $\rightarrow \mathrm{F} 1$ GVHD model. Recipients of MyD88KO TCD-BM plus WT T cells were injected with either $1.0 \times 10^{6} \mathrm{CD} 11 \mathrm{~b}^{+} \mathrm{Gr}-1^{+}$ MDSCs isolated from WT or MyD88KO mice at days 3, 5, and 7 post transplantation. Individual data from two independent experiments described in Figure 4 were evaluated. Data are presented as means \pm s.e.m. (d) Information regarding the transplants recruited to the clinical study is shown and ratios of T cells/lineage-HLA-DR ${ }^{-}$CD $11 \mathrm{~b}^{+} \mathrm{CD}_{3} 3^{+}$MDSCs in PBMCs concerning the development of $\geqslant$grade 2 intestinal GVHD are plotted at engraftment for 55 patients who underwent allo-HSCT with a myeloablative conditioning regimen. Patient characteristics are detailed in Supplementary Table 1. ANC; absolute neutrophil count; BMT, bone marrow transplantation; GVHD, graft-vs.-host disease; KO, knockout; MDSC, myeloid-derived suppressor cell; TCD-BM, T-cell depleted bone marrow; WT, wild type.

cells, a lack of MyD88 specifically in host APCs did not alleviate GVHD even partially. ${ }^{12}$ Such findings suggest that (in contrast to the known role played by MyD88 as an instigator of the pro-inflammatory immune response) a major effect of MyD88 signaling in GVHD is a limitation of inflammation, rather than aggravation of GVHD via APC activation. Our results suggest that MyD88 signaling has a protective role in GVHD development, especially intestinal GVHD, mediating MDSC expansion and function. Similarly, extent of MDSC expansion was reduced in MyD88-deficient mice after cecal ligation and puncture in a model of sepsis ${ }^{13}$ and MyD88-deficient mice developed more severe intestinal inflammation than WT animals in an experimental model of inflammatory bowel disease, ${ }^{6}$ indicating the importance of MyD88 signaling in terms of protecting the host from development of several inflammatory diseases involving the intestine.
However, in an apparent contradiction of results supporting the suppressive role of MyD88 in intestinal inflammatory disease, an association of high LPS concentrations with severe GVHD has also been reported in numerous studies. ${ }^{2,8,29,30}$ Therefore, it is probable that both pro-inflammatory and regulatory effects are exerted by MyD88-mediated signaling during the overall development of GVHD pathophysiology, and that MDSC expansion may have a fundamental role in the inflammatory signaling component of the host innate immune response to TLR ligation. However, TLRs are known to be expressed in various types of cells and at variable levels within the intestine, and deliver different signals depending upon their location in the intestinal epithelium. ${ }^{31}$ In addition, B-cellintrinsic MyD88 signaling has been reported to protect against experimental inflammatory disease. ${ }^{32}$ Therefore, it is also probable that MyD88-mediated signaling may exert proinflammatory or regulatory effects in a cell-type-specific 
manner. Thus, any protective role by MyD88 may be associated with MDSC expansion in the context of GVHD, whereas the pro-inflammatory role may have been principally in intestinal epithelial cells. Overall, we suggest that stimulation of MyD88 signaling in donor BM increases the level of MDSCs, whereas blocking of such signaling in a recipient, may be an ideal strategy to selectively promote the positive role of MyD88 signaling and protect a host from the development of severe GVHD. ${ }^{4}$

Our results showing the importance of the immunosuppressive function of MDSCs in terms of prevention of early fatal GVHD are in agreement with those of previous reports, which proposed a link between the immunosuppressive properties of MDSCs and the extent of GVHD protection after experimental allo-HSCT. ${ }^{18,19}$ Just as injection of MDSCs derived from embryonic stem cells ${ }^{33}$ or generated ex vivo by IL-13 treatment ${ }^{34}$ reduced the lethality of GVHD, supplementation of WT MDSC (Figure 4) or transplantation with TCD-BM in which MDSC population was augmented (Figure 5) also enhanced GVHD survival in our experiments. The report of Wang et al. ${ }^{18}$ showing aggravation of GVHD upon MDSCremoval, and suppression of T-cell expansion and GVHD by adding functional MDSCs purified from spleens is consistent with our results. However, the cited authors claimed that injection of immature BM-MDSCs did not exert such suppressive effects. We consider that this discrepancy in terms of the capability of BM-MDSCs to ameliorate GVHD is attributable to differences in the experimental schedules of MDSC supplementation between the two studies, as exertion of a suppressive effect by MDSCs is flexible, depending on the relative abundance of MDSCs compared to T cells. Wang et al. administered BM-MDSCs once, via co-transplantation at the time of allo-HSCT, whereas we injected BM-MDSCs several times (at days 3, 5, and 7 post transplantation). Therefore, differences in the timing, frequency, and total amount of supplementation may sufficiently influence the functions of such BM-MDSCs. Moreover, the use of different GVHD models (a B6-to-BALB.C model vs. a B6-to-B6DF1 model, respectively) may also have influenced the results. Together, we suggest that reciprocal application of the two supplementation schedules using the two different GVHD models would allow the capacity of BM-MDSCs to suppress GVHD to be evaluated more precisely. However, it is clear that MyD88-deficient MDSCs are defective in terms of ameliorating GVHD.

In the clinic, prediction of the likelihood of intestinal GVHD is critical from a therapeutic perspective. As gastrointestinal tract damage has a central role in amplifying systemic GVHD by propagating a cytokine storm, early and accurate diagnosis of the pathological condition is essential to allow appropriate therapeutic management of patients with acute GVHD. In this respect, the observed correlation between T/MDSC ratios in blood and the severity of intestinal GVHD may mean that the ratios are clinically useful surrogate markers of the condition, and signal the utility of a novel combined strategy to rescue gastrointestinal damage in the clinical context.
In summary, MyD88-dependent expansion of the CD11b ${ }^{+}$ Gr- $1^{+}$population from donor TCD-BM regulates the severity of GVHD by increasing apoptosis of and reducing intestinal infiltration by activated $\mathrm{T}$ cells. In addition, our data suggest that the existence of MyD88 signaling in the $\mathrm{CD} 11 \mathrm{~b}^{+} \mathrm{Gr}-1^{+}$ population is critical in terms of attenuation of the severity of GVHD early after transplantation. The results of our study afford an understanding of the mechanism by which MyD88mediated signaling in donor $\mathrm{BM}$ attenuates the severity of GVHD, and represents a critical step toward the potential clinical application of MDSCs.

\section{METHODS}

Mice. Female B6 $\left(\mathrm{H}-2^{\mathrm{b}}\right)$, B6.Ly-5a $\left(\mathrm{CD} 45.1^{+}\right)$, and F1 $\left(\mathrm{H}-2^{\mathrm{b} / \mathrm{d}}\right)$ mice were purchased from Japan SLC (Shizuoka, Japan). Female C.B10$\mathrm{H} 2^{\mathrm{b}} /$ LilMcdJ (BALB.B, H-2 ${ }^{\mathrm{b}}$ ) mice were purchased from the Jackson Laboratory (Bar Harbor, ME). MyD88-deficient (B6-MyD88 ${ }^{-1-}$, $\mathrm{H}-2^{\mathrm{b}}$ ) mice were generated by Kawai et al. ${ }^{35}$ and had been backcrossed $>10$ generations onto the $\mathrm{C} 57 \mathrm{BL} / 6 \mathrm{~J}$ strain.

Patients. Of the 131 patients consecutively transplanted in our institute from January through December 2013, 55 were subjected to myeloablative conditioning regimens (Supplementary Table 1). All the studies with humans were performed with approval from the Institutional Review Board (KC12SISE0585) of our institute in accordance with the Declaration of Helsinki.

Human MDSC analysis. Fresh peripheral blood mononuclear cells of patients undergoing allo-HSCT were retrieved at engraftment and were co-stained with FITC-conjugated anti-HLA-DR, PerCP-Cy5.5conjugated anti-Lineage, V450-conjugated anti-CD33, APC-Cy7conjugated anti-CD11b, PE-CF594-conjugated anti-CD15, and APC-conjugated anti-CD14 (BD Pharmingen, San Diego, CA) to determine their MDSC phenotype. For the frequency of the MDSC subsets, at least $1 \times 10^{5}$ events were acquired for analysis.

Induction of acute GVHD experimental allo-HSCT and assessment of GVHD. Mice underwent transplantation using a standard protocol described previously. ${ }^{36,37}$ Briefly, B6D2F1 recipients received TCD$\mathrm{BM}$ cells $\left(5 \times 10^{6}\right)$ plus $1 \times 10^{6}$ purified T cells from allogeneic B6 mice after total body irradiation with $1,100 \mathrm{cGy}$. B6.Ly-5a $\left(\mathrm{CD} 45.1^{+}\right)$mice were used to identify donor $\mathrm{T}$ cells in various organs. In the miHAmismatched murine model of GVHD, BALB.B recipient mice received $900 \mathrm{cGy}$ total body irradiation in two doses separated by $5 \mathrm{~h}$. Next, TCD-BM cells $\left(5 \times 10^{6}\right)$ and T cells $\left(5 \times 10^{6}\right)$ purified from B6 mice were injected into recipients. The extent of systemic acute GVHD was assessed using a scoring system that incorporates five clinical parameters. ${ }^{38}$ The histological grading criteria of intestinal GVHD were as follows: grade 0 , normal; grade 1 , single-crypt apoptosis; grade 2 , loss of a crypt; grade 3 , dropout of two or more continuous crypts; grade 4, no identifiable residual viable crypts (total epithelial denudation). ${ }^{39}$

Flow cytometric analysis and assessment of donor cell apoptosis. Single-cell suspensions were stained in FACS buffer at $4{ }^{\circ} \mathrm{C}$ for $30 \mathrm{~min}$. In vivo apoptosis of donor T cells was analyzed by flow cytometry, as described previously. ${ }^{40}$ Samples were analyzed using a FACSCalibur and an LSRII (BD Pharmingen). The antibodies used in the analyses are described in Supplementary Data. The following antibodies against mouse antigens were purchased from BD Pharmingen: FITCconjugated anti-H- ${ }^{\mathrm{d}}$, anti-CD45.1, anti-c-kit, anti-CD11c, anti-CD8, anti-IL-1 $\beta$, and anti-TNF $\alpha$; PE-conjugated anti-CD11b, anti-sca-1, anti-Ly6C and anti-CD4; PerCP-Cy5.5-conjugated anti-CD8; APCconjugated anti-B220, anti-Ly6G (Gr-1), anti-Foxp3, anti-IFN $\gamma$, antiBrdU, Annexin V, and CCR9; and APC-Cy7-conjugated anti-CD4. $\mathrm{PE}-\mathrm{Cy} 7-\mathrm{conjugated} \mathrm{anti-F4/80} \mathrm{was} \mathrm{purchased} \mathrm{from} \mathrm{Miltenyi} \mathrm{Biotec}$ 
(Auburn, CA). FITC-conjugated anti-caspase 9 (E23) and anti-BAX (E63) were purchased from Abcam (Cambrige, UK).

In vivo proliferation (BrdU incorporation). Mice were treated with two doses of BrdU ( 2 mg per mouse; Koram Biotech Corp, Seoul, Korea) for $4 \mathrm{~h}$ in total on day 4 post transplantation, and were killed $2 \mathrm{~h}$ after the second dose to obtain splenocytes. The splenocytes were stained with CD45.1-FITC, anti-CD4- APC-Cy7, and anti-CD8- PerCP-Cy5.5.

Histologic and confocal microscopic analyses. Formalin-fixed, paraffin-embedded tissue sections were stained with hematoxylin and eosin. Cryosections were incubated with anti-CD45.1-PE, and, after washing, counterstained with 4,6-diamidino-2-phenylindole (DAPI; Sigma-Aldrich, St Louis, MO). Slides were examined using a confocal microscope (LSM700; Carl Zeiss, Oberkochen, Germany).

Isolation of MDSCs from donor BM. BM cells were incubated with an FcR-blocking reagent for $10 \mathrm{~min}$ and stained with biotin-conjugated anti-Ly6G, followed by positive magnetic selection of MDSCs using anti-biotin microbeads, according to the manufacturer's instructions (Miltenyi Biotec).

Mononuclear cell isolation from the intestine. Mononuclear cells infiltrating the intestine were isolated using a method described in a previous report. ${ }^{41}$ In brief, intestinal specimens (1-cm-long pieces) were treated with $0.75 \mathrm{mM}$ EDTA and collagenase, and next centrifuged after overlaying on Ficoll-Paque solution. Mononuclear cells were recovered from the interface.

LPS administration. Mice were subjected to intraperitoneal injection of LPS from Escherichia coli (O128-B12; Sigma-Aldrich) at $2 \mathrm{mg} \mathrm{kg}^{-1}$ daily for 3 days. Control mice received equal amounts of the LPS vehicle (saline intraperitoneal).

RT-PCR analyses. To detect MyD88 mRNA expression, PCR was performed using MyD88-specific primers (sense: $5^{\prime}$-TGGCATGCC TCCATCATAGTTAACC- $3^{\prime}$ and antisense $5^{\prime}$-GTCAGAAACAA CCACCACCATGC-3'). PCR amplification featured 28 cycles of denaturation at $94^{\circ} \mathrm{C}$ for $30 \mathrm{~s}$, annealing at $55^{\circ} \mathrm{C}$ for $60 \mathrm{~s}$, and elongation at $72{ }^{\circ} \mathrm{C}$ for $30 \mathrm{~s}$. To detect Arg-1 and iNOS mRNA expression, real-time quantitative PCR was performed using an SYBR Green Master Mix and run in a CFX96 real-time thermal cycler (Bio-Rad, Hercules, CA). The following primers were used: murine Arg-1 primers: forward, 5'-CTCCAAGCCAAAGTCCTTAGAG-3', and reverse, $5^{\prime}$ - AGGAGCTGTCATTAGGGACATC-3'; murine iNOS primers: forward, 5'-GTTCTCAGCCCAACAATACAAGA-3', and reverse, $5^{\prime}$-GTGGACGGGTCGATGTCAC-3'; murine GAPDH primers: forward, 5'-GGTGTGAACGGATTTGGCCGTATT-3', and reverse, 5'-GGCCTTGACTGTGCCGTTGAATTT-3'.

Cytometric bead analysis. The concentrations of six cytokines (IFN$\gamma$, TNF- $\alpha$, IL-1 $\beta$, IL-6, IL-17A, and IL-10) in recipient sera were determined using a commercially available kit (BD Pharmingen). All the tests were performed according to the manufacturer's instructions.

Protein extraction and measurement of chemokine levels by enzyme-linked immunosorbent assay. Tissue samples were homogenized in 2-ml volumes of buffer. Supernatants were harvested after centrifugation and the level of CCL25 was measured using a kit (R\&D Systems, Minneapolis, MN) according to the manufacturer's protocol.

Statistical procedures. All the values are expressed as means \pm s.e.m. Comparisons between groups were performed using the parametric independent samples $t$-test if the groups contained more than five animals, or using the Mann-Whitney test otherwise. The Wilcoxon rank-sum test was used to analyze the survival data.

SUPPLEMENTARY MATERIAL is linked to the online version of the paper at http://www.nature.com/mi

\section{ACKNOWLEDGMENTS}

This study was supported by the Korea Healthcare Technology R\&D Project, Ministry of Health, Welfare, and Family Affairs, Republic of Korea (Grant no. A120262) and a grant from the National Research Foundation of Korea (Science Research Center 2014051826).

\section{AUTHOR CONTRIBUTIONS}

J.-Y.L., Y.-K.L., and J.-M.J. performed the experiments, collected and analyzed the data, and participated in writing the manuscript; S.-E.L. helped write the manuscript; G.P. was responsible for histopathology; K.-S.E., Y.-J.K., N.-G.C., and D.C.J. supervised all the laboratory research; E.Y.C. and C.-K.M. designed the research, analyzed and interpreted the data, and wrote the manuscript.

\section{DISCLOSURE}

The authors declared no conflict of interest.

(c) 2016 Society for Mucosal Immunology

\section{REFERENCES}

1. Ferrara, J.L., Levine, J.E., Reddy, P. \& Holler, E. Graft-versus-host disease. Lancet 373, 1550-1561 (2009).

2. Hill, G.R., Crawford, J.M., Cooke, K.R., Brinson, Y.S., Pan, L. \& Ferrara, J.L. Total body irradiation and acute graft-versus-host disease: the role of gastrointestinal damage and inflammatory cytokines. Blood 90, 32043213 (1997).

3. Caron, G. et al. Direct stimulation of human T cells via TLR5 and TLR7/8: flagellin and R-848 up-regulate proliferation and IFN-gamma production by memory CD4 + T cells. J. Immunol. 175, 1551-1557 (2005).

4. Heimesaat, M.M. et al. MyD88/TLR9 mediated immunopathology and gut microbiota dynamics in a novel murine model of intestinal graft-versus-host disease. Gut 59, 1079-1087 (2010).

5. Quigley, M., Martinez, J., Huang, X.\& Yang, Y. A critical role for direct TLR2MyD88 signaling in CD8 T-cell clonal expansion and memory formation following vaccinia viral infection. Blood 113, 2256-2264 (2009).

6. Rakoff-Nahoum, S., Paglino, J., Eslami-Varzaneh, F., Edberg, S. \& Medzhitov, R. Recognition of commensal microflora by toll-like receptors is required for intestinal homeostasis. Cell 118, 229-241 (2004).

7. Hill, G.R. \& Ferrara, J.L. The primacy of the gastrointestinal tract as a target organ of acute graft-versus-host disease: rationale for the use of cytokine shields in allogeneic bone marrow transplantation. Blood 95, 2754-2759 (2000).

8. Cooke, K.R. et al. LPS antagonism reduces graft-versus-host disease and preserves graft-versus-leukemia activity after experimental bone marrow transplantation. J. Clin. Invest. 107, 1581-1589 (2001).

9. Elmaagacli, A.H. et al. Mutations in innate immune system NOD2/CARD 15 and TLR-4 (Thr399lle) genes influence the risk for severe acute graft-versus-host disease in patients who underwent an allogeneic transplantation. Transplantation 81, 247-254 (2006).

10. Taylor, P.A. et al. TLR agonists regulate alloresponses and uncover a critical role for donor APCs in allogeneic bone marrow rejection. Blood 112, 3508-3516 (2008).

11. Shlomchik, W.D.J. et al. Prevention of graft versus host disease by inactivation of host antigen-presenting cells. Science 285, 412-415 (1999).

12. Li, H. et al. Graft-versus-host disease is independent of innate signaling pathways triggered by pathogens in host hematopoietic cells. J. Immunol. 186, 230-241 (2011).

13. Delano, M.J. et al. MyD88-dependent expansion of an immature GR$1(+) \mathrm{CD} 11 \mathrm{~b}(+)$ population induces T cell suppression and Th2 polarization in sepsis. J. Exp. Med. 204, 1463-1474 (2007).

14. Cheng, L. et al. Interleukin- 6 induces Gr-1 $+\mathrm{CD} 11 \mathrm{~b}+$ myeloid cells to suppress CD8 + Tcell-mediated liver injury in mice. PLoS One 6, e17631 (2011).

15. Melani, C., Sangaletti, S., Barazzetta, F.M., Werb, Z. \& Colombo, M.P. Amino-biphosphonate-mediated MMP-9 inhibition breaks the tumor-bone marrow axis responsible for myeloid-derived suppressor cell expansion and macrophage infiltration in tumor stroma. Cancer Res. 67, 1143811446 (2007). 
16. Kujawski, M., Kortylewski, M., Lee, H., Herrmann, A., Kay, H. \& Yu, H. Stat3 mediates myeloid cell-dependent tumor angiogenesis in mice. J. Clin. Invest. 118, 3367-3377 (2008).

17. Nagaraj, S. \& Gabrilovich, D.I. Myeloid-derived suppressor cells. Adv. Exp. Med. Biol. 601, 213-223 (2007).

18. Wang, D. etal. Dynamic change and impact of myeloid-derived suppressor cells in allogeneic bone marrow transplantation in mice. Biol. Blood Marrow Transplant. 19, 692-702 (2013).

19. Ghansah, T. et al. Expansion of myeloid suppressor cells in SHIP-deficient mice represses allogeneic T cell responses. J. Immunol. 173, 7324-7330 (2004).

20. Lim, J.Y., Choi, M.S., Youn, H., Choi, E.Y. \& Min, C.K. The influence of pretransplantation conditioning on graft-vs.-leukemia effect in mice. Exp. Hematol. 39, 1018-1029 (2011).

21. Chen, X., Dodge, J., Komorowski, R. \& Drobyski, W.R. A critical role for the retinoic acid signaling pathway in the pathophysiology of gastrointestinal graft-versus-host disease. Blood 121, 3970-3980 (2013).

22. Zhang, Y. et al. APCs in the liver and spleen recruit activated allogeneic CD8 + Tcells to elicit hepatic graft-versus-host disease. J. Immunol. 169, 7111-7118 (2002).

23. Youn, J.I., Collazo, M., Shalova, I.N., Biswas, S.K. \& Gabrilovich, D.I. Characterization of the nature of granulocytic myeloid-derived suppressor cells in tumor-bearing mice. J. Leuk. Biol. 91, 167-181 (2012).

24. Xia, S., Sha, H., Yang, L., Ji, Y., Ostrand-Rosenberg, S. \& Qi, L. Gr-1+ $\mathrm{CD11b}+$ myeloid-derived suppressor cells suppress inflammation and promote insulin sensitivity in obesity. J. Biol. Chem. 286, 23591-23599 (2011).

25. Choi, E.Y. et al. Real-time T-cell profiling identifies H60 as a major minor histocompatibility antigen in murine graft-versus-host disease. Blood 100, 4259-4265 (2002).

26. De Wilde, V. et al. Endotoxin-induced myeloid-derived suppressor cells inhibit alloimmune responses via heme oxygenase-1. Am. J. Transplant. 9 , 2034-2047 (2009).

27. Ostrand-Rosenberg, S. \& Sinha, P. Myeloid-derived suppressor cells: linking inflammation and cancer. J. Immunol. 2009)182, 4499-4506.

28. Matte, C.C. et al. Donor APCs are required for maximal GVHD but not for GVL. Nat. Med. 10, 987-992 (2004).

29. Cooke, K.R. et al. Tumor necrosis factor- alpha production to lipopolysaccharide stimulation by donor cells predicts the severity of experimental acute graft-versus-host disease. J. Clin. Invest. 102, 1882-1891 (1998).
30. Nestel, F.P., Price, K.S., Seemayer, T.A. \& Lapp, W.S. Macrophage priming and lipopolysaccharide-triggered release of tumor necrosis factor alpha during graft-versus-host disease. J. Exp. Med. 175, 405-413 (1992).

31. Lee, J. et al. Maintenance of colonic homeostasis by distinctive apical TLR9 signalling in intestinal epithelial cells. Nat. Cell Biol. 8, 1327-1336 (2006).

32. Kirkland, D. et al. B cell-intrinsic MyD88 signaling prevents the lethal dissemination of commensal bacteria during colonic damage. Immunity 36, 228-238 (2012).

33. Zhou, Z. et al. Development and function of myeloid-derived suppressor cells generated from mouse embryonic and hematopoietic stem cells. Stem Cells 28, 620-632 (2010).

34. Highfill, S.L. et al. Bone marrow myeloid-derived suppressor cells (MDSCs) inhibit graft-versus-host disease (GVHD) via an arginase-1-dependent mechanism that is up-regulated by interleukin-13. Blood 116, 5738-5747 (2010).

35. Kawai, T., Adachi, O., Ogawa, T., Takeda, K. \& Akira, S. Unresponsiveness of MyD88-deficient mice to endotoxin. Immunity 11, 115-122 (1999).

36. Min, C.K. et al. Paradoxical effects of interleukin- 18 on the severity of acute graft-versus-host disease mediated by CD4 + and CD8 + T-cell subsets after experimental allogeneic bone marrow transplantation. Blood 104, 3393-3399 (2004).

37. Min, C.K., Kim, B.G., Park, G., Cho, B. \& Oh, I.H. IL-10-transduced bone marrow mesenchymal stem cells can attenuate the severity of acute graftversus-host disease after experimental allogeneic stem cell transplantation. Bone Marrow Transplant. 39, 637-645 (2007).

38. Cooke, K.R. et al. An experimental model of idiopathic pneumonia syndrome after bone marrow transplantation: I. The roles of minor $\mathrm{H}$ antigens and endotoxin. Blood 88, 3230-3239 (1996).

39. Cheung, D.Y. et al. Endoscopic evaluation in gastrointestinal graft-versushost disease: comparisons with histological findings. Dig. Dis. Sci. 53, 2947-2954 (2008).

40. Svensson, M. et al. CCL25 mediates the localization of recently activated CD8alphabeta( + ) lymphocytes to the small-intestinal mucosa. J. Clin. Invest. 110, 1113-1121 (2002).

41. Comer, G.M., Ramey, W.G., Kotler, D.P. \& Holt, P.R. Isolation of intestinal mononuclear cells from colonoscopic biopsies for immunofluorescence analysis by flow cytometry. Dig. Dis. Sci. 31, 151-156 (1986). 